Originalveröffentlichung in: Guthmüller, Bodo ; Kühlmann, Wilhelm (Hrsgg.): Renaissancekultur und antike Mythologie, Tübingen 1999, S. 253-281 (Frühe Neuzeit ; 50)

\author{
Ulrich Rehm
}

\title{
Instaurare iubet tunc hymenaea Venus: Botticellis Primavera
}

The Pleasure that Painting, as a Dumb Art, gives us, is like what we have from Musick; its beautiful Forms, Colours and Harmony, are to the Eye what Sounds, and the Harmony of that kind are to the Ear ${ }^{1}$

Kaum einem zweiten Gemälde wurden in den letzten über hundert Jahren Kunstgeschichtsschreibung so zahlreiche und unterschiedliche Deutungen zuteil wie der sogenannten Primavera (Abb. 1) von Alessandro Felipepi, der unter dem Namen Sandro Botticelli bekannt ist. ${ }^{2} 1893$ erschien Aby Warburgs grundlegende Deutung ${ }^{3}$ zu dieser Frühlingsallegorie und der $G e$ burt der Venus ${ }^{4}$ Erst seit 1975 müssen sich die Interpretationen der Primavera an der genaueren Kenntnis ihres Entstehungszusammenhangs messen lassen: ${ }^{5}$ Damals zeigten John Shearman und Webster Smith, daß das Bild

1 Jonathan Richardson: An Essay on the Theory of Painting. London 1715, S. 5.

2 Der Text führt einen vom Verf. bereits in einer Rezension angedeuteten Interpretationsvorschlag aus (vgl. Kunstchronik 47 [1994] S. 96-104). Anregungen werden dem Arbeitsgespräch »Renaissancekultur und klassische Mythologie« (9. Oktober 1996) sowie dem Bonner Kolloquium (281. Sitzung, 13. Januar 1997) verdankt. - Zu Leben und Werk Botticellis zusammenfassend: Charles Dempsey: Botticelli, Sandro. In: The Dictionary of Art. Bd. 4. New York 1996, S. 493-504. - Norberto Massi: Botticelli. In: Saur. Allgemeines Künstlerlexikon. Die Bildenden Künstler aller Zeiten und Völker. Bd. 13. München und Leipzig 1996, S. 261-264.

3 Aby Warburg: Sandro Botticelli's »Geburt der Venus« und »Frühling«. Hamburg 1893; wiederabgedruckt in: Ders.: Gesammelte Schriften. Die Erneuerung der heidnischen Antike. Hg. von Gertrud Bing. Bd. 1. Leipzig und Berlin 1932, S. 5-68 und 307-328. Gemälde auf Leinwand $(172,5$ x 278,5 cm). Florenz, Galleria degli Uffizi.

5 Zu früheren Deutungen vgl. zum Beispiel: Ernst H. Gombrich: Botticelli's Mythologies. A study in the neoplatonic symbolism of his circle. In: Journal of the Warburg and Courtauld Institutes 8 (1945), S. 7-60; wiederabgedruckt in: Ders.: Symbolic images. Studies in the art of the renaissance. London 1972, S. 31-81. - Pierre Francastel: La fête mythologique au Quattrocento. Expression littéraire et visualisation plastique. In: Revue d'esthétique 6 (1952), S. 376-410; wiederabgedruckt in: Ders.: CEuvres II. La réalité figurative. Éléments structurels de sociologie et de l'art. Paris 1965, S. 229-252. - Pietro Maria Bardi: La Primavera. Mailand 1953. - Arnolfo B. Ferruolo: Botticelli's mythologies, Ficino's ,de amore<, Poliziano's sstanze per la giostra<. Their circle of love. In: Art Bulletin 37 (1955), S. 17-25. - Pierre Francastel: Un mito poetico y social de Quattrocento: La Primavera. In: La totte. Revista general de la Universidad de Puerto Rico 5 (1957), S. 23-41; wiederabgedruckt in franz. Übersetzung: Un mythe poétique et social du Quattrocento: La Primavera. In: EEuvres II. La réalité figurative. Éléments structurels de sociologie et de l'art. Paris 1965, S. 253-266. - Edgar Wind: Pagan mysteries in the renaissance. New Haven 1958, S. 100-120. - Erwin Panofsky: Renaissance and renascences in Western art. Uppsala 1960, S. 191-200. - Luisa Becherucci: Botticelli. La Primavera. Florenz 1965 (Forma e colore 53). - Charles Dempsey: sMercurius Ver<. The sources of Botticelli's PPrimavera<. In: Journal of the Warburg and Courtauld Institutes 31 (1968), S. 251-273. - 
wahrscheinlich nicht ursprünglich - wie bis dahin vermutet - für den MediciLandsitz in Castello bestimmt war. Dort hatte im 16. Jahrhundert Giorgio Vasari das Gemälde gesehen. ${ }^{6}$ Die Auswertung eines Inventars von 1499 im Florentiner Staatsarchiv legte vielmehr nahe, daß das Gemälde zunächst zur Ausstattung der case vecchie in der Via Larga in Florenz gehörte (heute Via Cavour). ${ }^{7}$ Diese case wurden von einem Vetter des sil Magnificor genannten Lorenzo de' Medici bewohnt, von Lorenzo di Pierfrancesco de' Medici, der somit als wahrscheinlicher Auftraggeber der Primavera gelten darf.

\section{I. apicato sopra el letucio}

In dem vor dessen Schlafzimmer gelegenen Raum $^{8}$ war eine mit der Primavera identifizierte Tafel 1499 an ihrem wohl ursprünglichen Bestimmungsort angebracht, und zwar als Bestandteil eines Möbelstücks, am lettuccio. ${ }^{9}$ Ein zeitgenössischer Florentiner Holzschnitt (Abb. 2) zeigt ein solches Möbel. Die Stelle, die hier eine intarsiengeschmückte Rückwand einnimmt, war am lettuccio im Hause Lorenzos Botticellis Gemälde vorbehalten. Dies mag die für ein Tafelbild nicht gerade gewöhnlichen Maße von 203 x 314 $\mathrm{cm}$ erklären. Die Breite des Gemäldes liegt knapp unter der im Inventar angegebenen Länge des lettuccio.

Die Vermutung Warburgs, die Primavera sei als Pendant zur Geburt der Venus geschaffen worden, war somit hinfällig. Zwar hatte dieser Gedanke dadurch nahegelegen, daß Vasari beide Bilder in Castello erwähnt hatte und daß beide Venus als zentrale Gestalt zeigen; im Inventar von 1499 wird allerdings ein anderes Gemälde erwähnt, das im selben Raum über der Tür zum Vorzimmer hing. Dieses wird - überzeugend - mit Botticellis Bild mit Minerva (oder Camilla) und Kentaur (Abb. 3) identifiziert. ${ }^{10}$

Charles Dempsey: Botticelli's three graces. In: Journal of the Warburg and Courtauld Institutes 34 (1971), S. 326-330.

6 »... oggi ancora a Castello, villa del duca Cosimo, sono due quadri figurati l'uno Venere che nasce, e quelle aure e venti che la fanno venire in terra con gli Amori, e così un'altra Venere che le Grazie la fioriscono, dinotando la Primavera, le quali da lui con grazia si veggono espresse « (Giorgio Vasari: Le vite de' più eccellenti pittori scultori e architettori nelle redazioni del 1550 e 1568 . Testo a cura di Rosanna Bettarini. Commento secolare a cura di Paola Barocchi. Bd. 3. Florenz 1971, S. 513-514).

7 John Shearman: The collections of the younger branch of the Medici. In: Burlington Magazine 117 (1975), S. 12-27; Webster Smith: On the original location of the Primavera. In: Art Bulletin 57 (1975), S. 31-39.

8 "Siegue in la Camara Terena alato ala Camara di Lorenzo (Florenz, Archivio di Stato, Archivio mediceo avanti il principato, filza CXXIX, fol. 517r; zitiert nach Smith, S. 37).

9 »Uno quadro di lignamo apicato sopra el letucio nel quale e dipinto nove figure di donne e homini estimat L 100 « (Florenz, Archivio di Stato, Archivio mediceo avanti il principato, filza CXXIX, fol. 517r; zitiert nach Smith, S. 37).

10 »Uno quadro di lignamo di supra lusso di lantichamara nel quale e dipinto chamilo (sic) con uno satilo (sic) est L 40 « (Florenz, Archivio di Stato, Archivio mediceo avanti il principato, filza CXXIX, fol. 517r; zitiert nach Smith, S. 38). 


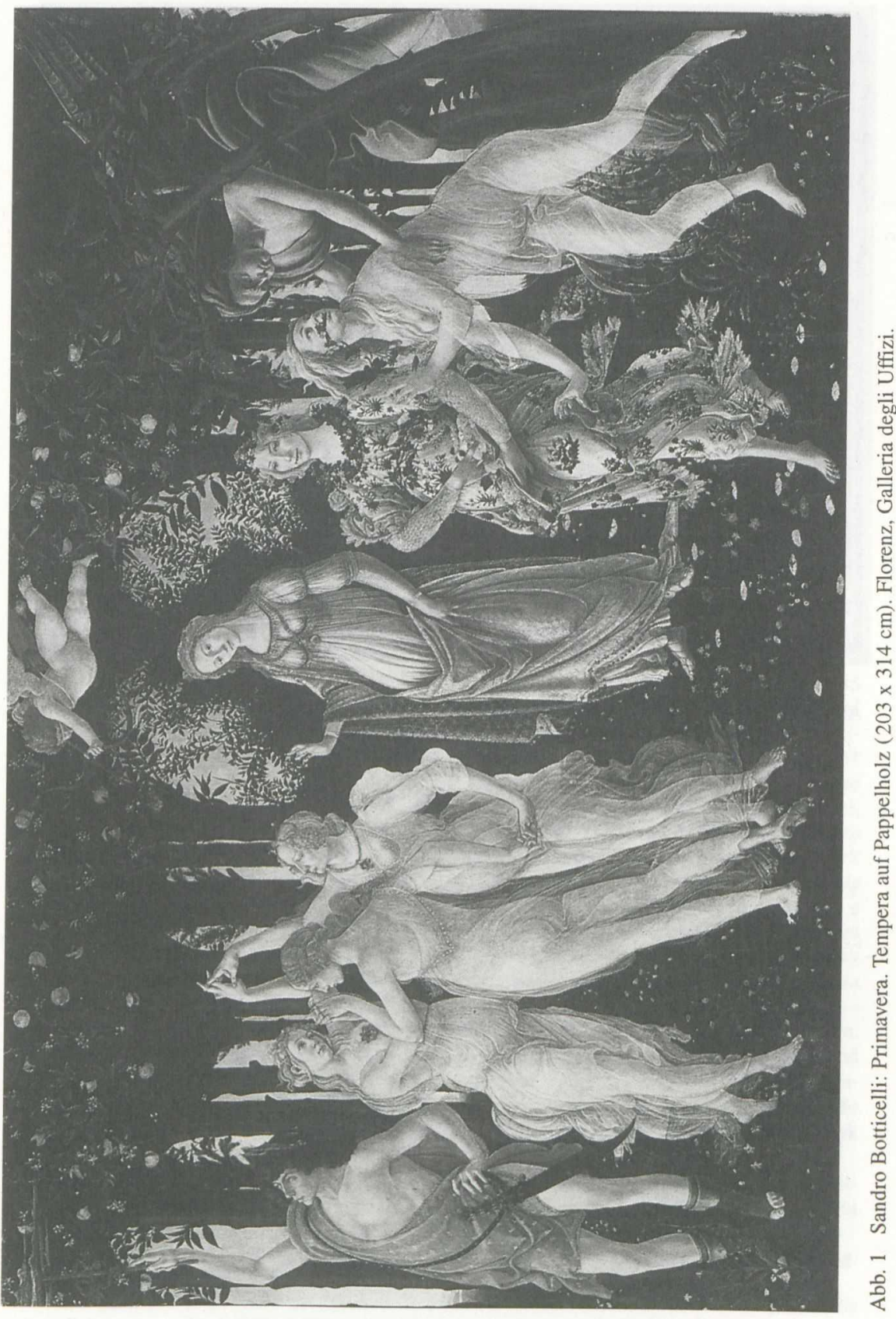




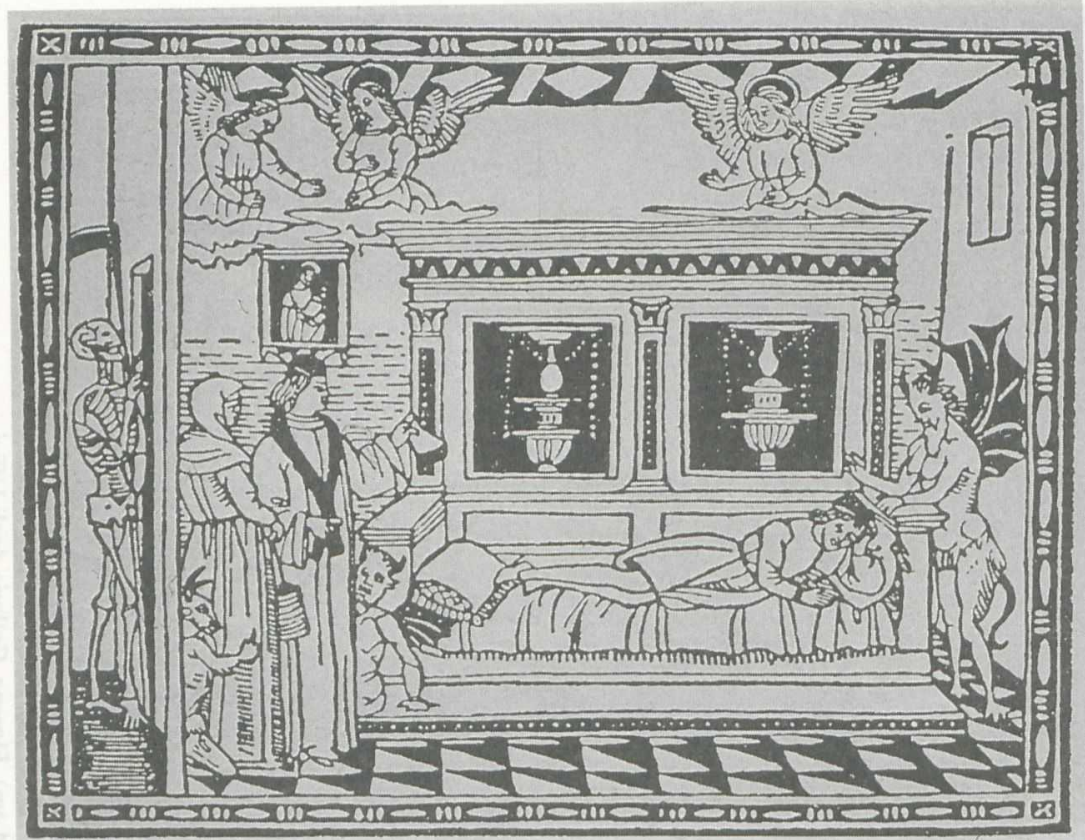

Abb. 2 Holzschnitt-Illustration zu Girolamo Savonarola: Predica dell'arte del bene morire. Florenz 1496/97. 
Merkwürdigerweise wurden für die Interpretation der Primavera aus diesen Kenntnissen kaum Konsequenzen gezogen. ${ }^{11}$ Horst Bredekamp deutete das Bild zwar 1990 als subtile politische Stellungnahme des PierfrancescoZweigs der Medici; allerdings bietet die Primavera keine so spezifischen Motive, daß sie sich nicht auch auf andere Mitglieder der Familie beziehen ließe. Zudem bleibt die Frage, ob ein lettuccio der angemessene Ort für ein komplexes politisches Bekenntnis sei. ${ }^{12}$ Einige Autoren, so Ronald Lightbown, äußerten die Vermutung, das Bild könne in Verbindung zur Hochzeit Lorenzo di Pierfrancescos gestanden haben. ${ }^{13}$ Erst Michael Rohlmann führte kürzlich in einem Vortrag aus, was mit der Entdeckung von 1975 im Grunde nahegelegt war: Der mit dem lettuccio ausgestattete Raum neben dem Schlafzimmer des Hausherrn war - damaligen Gepflogenheiten gemäß - wahrscheinlich das Gemach der Gemahlin Lorenzos, ${ }^{14}$ denn es war üblich, daß die Ehegatten getrennte Schlafzimmer bewohnten, die miteinander in Verbindung standen. Dementsprechend dürfte die Primavera zu der von Lorenzo di Pierfrancesco zur Hochzeit veranlaßten Ausstattung des Brautgemachs gehört haben, was durch die bisher am besten begründete Datierung in die frühen 80er Jahre - die Zeit kurz nach dem Romaufenthalt Botticellis - bestätigt wird. ${ }^{15}$ Am 19. Juli 1482 heiratete Lorenzo Semiramide

11 Zu den seither erschienenen Deutungen vgl. zum Beispiel: Paul Holberton: Botticelli's Primavera, che voleva intendesse. In: Journal of the Warburg and Courtauld Institutes 45 (1982), S. 202-210. - Mirella Levi D'Ancona: Botticelli's Primavera. A botanical interpretation including astrology, alchemy and the Medici. Florenz 1983. - Paul Barolsky: Botticelli's Primavera and the tradition of Dante. In: Kunsthistorisk Tidskrift 51 (1983), S. 1-16. - Umberto Baldini u. a.: La Primavera del Botticelli. Storia di un quadro e di un restauro. Mailand 1984 (mit Beiträgen von Ornella Casazza, Mauro Matteini, Guido Moggi, Arcangelo Moles und Maurizio Seracini). - Liana Cheney: Quattrocento Neoplatonism and Medici Humanism in Botticelli's Mythological Paintings. New York und London 1985. - Horst Bredekamp: Sandro Botticelli. La Primavera. Florenz als Garten der Venus. Frankfurt a. M. 1988. - Alessandro Parronchi: Botticelli fra Dante e Petrarca. Florenz 1985. - Charles Dempsey: The Portrayal of Love. Botticelli's Primavera and Humanist Culture at the Time of Lorenzo the Magnificent. Princeton, New Jersey 1992. - Mirella Levi D’Ancona: Due quadri del Botticelli eseguiti per nascite in Casa Medici. Nuova interpretazione della Primavera e della Nascita di Venere. Florenz 1992. Joanne Snow-Smith: The Primavera of Sandro Botticelli. A Neoplatonic Interpretation. New York 1993 (New Connections. Studies in Interdisciplinarity 5). - Paul Barolsky und Anne Barriault: Botticelli's Primavera and the origins of the elegiac in Italian Renaissance painting. In: Gazette des Beaux-Arts. 6e période, tom. 128, Jg. 138 (1996) S. 6370. - Charles Dempsey: Botticelli, Sandro. In: The Dictionary of Art. Bd. 4. New York 1996, S. 493-504 (zur Primavera S. 498f.).

12 Bredekamp: Primavera. - Charles Dempsey argumentierte in seinem eigens der Primavera gewidmeten Buch noch 1992 an den Dokumenten vorbei, indem er das Bild erneut als eine Art poetisches Vermächtnis des Magnifico interpretierte (Dempsey: Portrayal).

13 Ronald Lightbown: Sandro Botticelli. London 1989, hier benutzt: deutsche Übersetzung. München 1989, S. 142-143.

14 Erst nach Abfassen des vorliegenden Textes erschienen: Michael Rohlmann: Botticellis „Primavera«. Zu Anlaß, Adressat und Funktion von mythologischen Gemälden im Florentiner Quattrocento. In: artibus et historiae 32 (1996), S. 97-132. Vgl. auch W. A. Bulst: Die ursprüngliche innere Aufteilung des Palazzo Medici in Florenz. In: Mitteilungen des Kunsthistorischen Instituts in Florenz 14 (1970), S. 369-392, hier vor allem S. 376, Anm. 22. 
d'Appiano, nachdem die ursprünglich für den Monat Mai angesetzte Trauung wegen eines Todesfalls hatte verschoben werden müssen.

Das Brautgemach zur Hochzeit mit Bildern auszustatten, die - wie die Primavera und Minerva und Kentaur - exemplarisch oder allegorisch um die Themen der Liebe, der Schönheit, der Fruchtbarkeit und der weiblichen Tugenden kreisten, war weithin üblich. Man denke nur an die relativ zahlreich erhaltenen, geschmückten cassoni ${ }^{16}$ oder die spalliere genannten Vertäfelungen der Schlafgemächer. ${ }^{17}$ Aber auch Hochzeitskästchen zeigen verwandte Themen, wie zum Beispiel das 1421 von Giovanni di Siena bemalte im Louvre: Hier tanzt auf einer Frühlingswiese eine Dame zwischen zwei Musikerinnen, über ihnen thront Venus. ${ }^{18}$

Faßt man die Primavera ihrem ursprünglichen Kontext entsprechend als Brautzimmerschmuck auf, bleibt zunächst weiterhin ungeklärt, worin genau der Grundgedanke des Bildprogramms besteht und woher er stammt. Die Schwierigkeiten beginnen bereits bei der oft diskutierten Frage, wie es kommt, daß Venus und Amor, Merkur, die drei Grazien, Flora, Chloris und Zephyr gemeinsam in einem Bild auftreten. Die ungewöhnliche Zusammenstellung scheint in besonderer Weise nach dem zu verlangen, was Leon Battista Alberti in Anlehnung an die Rhetorik als inventio bezeichnete, um so die Arbeitsschritte der Historienmalerei mit denen einer ars libera zu vergleichen. ${ }^{19}$ Eine literarische Quelle für die Primavera war bisher nicht ausfindig zu machen. So behalf man sich seit Aby Warburg mit einer recht komplizierten Zusammenstückung von Aussagen aus verschiedenen römischen Klassikern: Stellen aus Lucretius' De natura rerum, Ovids Fasti, Horaz' Carmina und Senecas De beneficiis wurden miteinander verknüpft und durch Dichtungen des Quattrocento ergänzt, besonders durch solche von Angelo Poliziano (1454-1494) und Lorenzo de' Medici. ${ }^{20}$ Als Gipfel dieser Entwicklung darf Charles Dempseys Versuch von 1992 gelten, den Mangel einer einheitlichen Quelle dadurch zu beheben, daß er eine Fülle ganz unterschiedlicher Texte unter dem Kunstbegriff »Scriptores rerum rusticarum $\ll^{21}$ zusammenfaßte. Dennoch blieb auch Dempseys Deutung defizitär,

15 Das Studium bestimmter in Rom aufbewahrter antiker Bildwerke als Voraussetzung für die Primavera zum Beispiel vertritt Henning Wrede: Der Antikengarten der del Bufalo bei der Fontana Trevi. In: 4. Trierer Winckelmannsprogramm 1982, S. $17 \mathrm{f}$.

16 Vgl. zum Beispiel Paul Schubring: Cassoni. Truhen und Truhenbilder der italienischen Frührenaissance, Bd. 1-2, Leipzig ${ }^{2} 1923$.

17 Vgl. dazu Lightbown: Botticelli, S. 114.

18 Paris, Louvre, Inv. nr. OA 2517. Vgl. auch P. F. Watson: The Garden of Love in Tuscan Art of the Early Renaissance. Philadelphia und London 1979.

19 Leon Battista Alberti: On Painting and On Sculpture. The Latin texts of De Pictura and De Statua. Edited with translations, introductions and notes by Cecil Grayson. London 1972, vgl. vor allem Liber III, 53, S. 95.

$20 \mathrm{Zu}$ Poliziano vgl. den jüngst erschienenen Artikel von Nicole Hegener: »Angelus Politianus enormi fuit naso, ...«. In: Antiquarische Gelehrsamkeit und Bildende Kunst. Die Gegenwart der Antike in der Renaissance. Köln 1996 (Atlas. Bonner Beiträge zur Renaissanceforschung. Hg. von Gunter Schweikhart, Bd. 1), S. 85-121.

21 So der Titel der 1472 von Columella herausgegebenen Texte Lucretius', Catos und Varros (Dempsey: >Mercurius Ver`). 


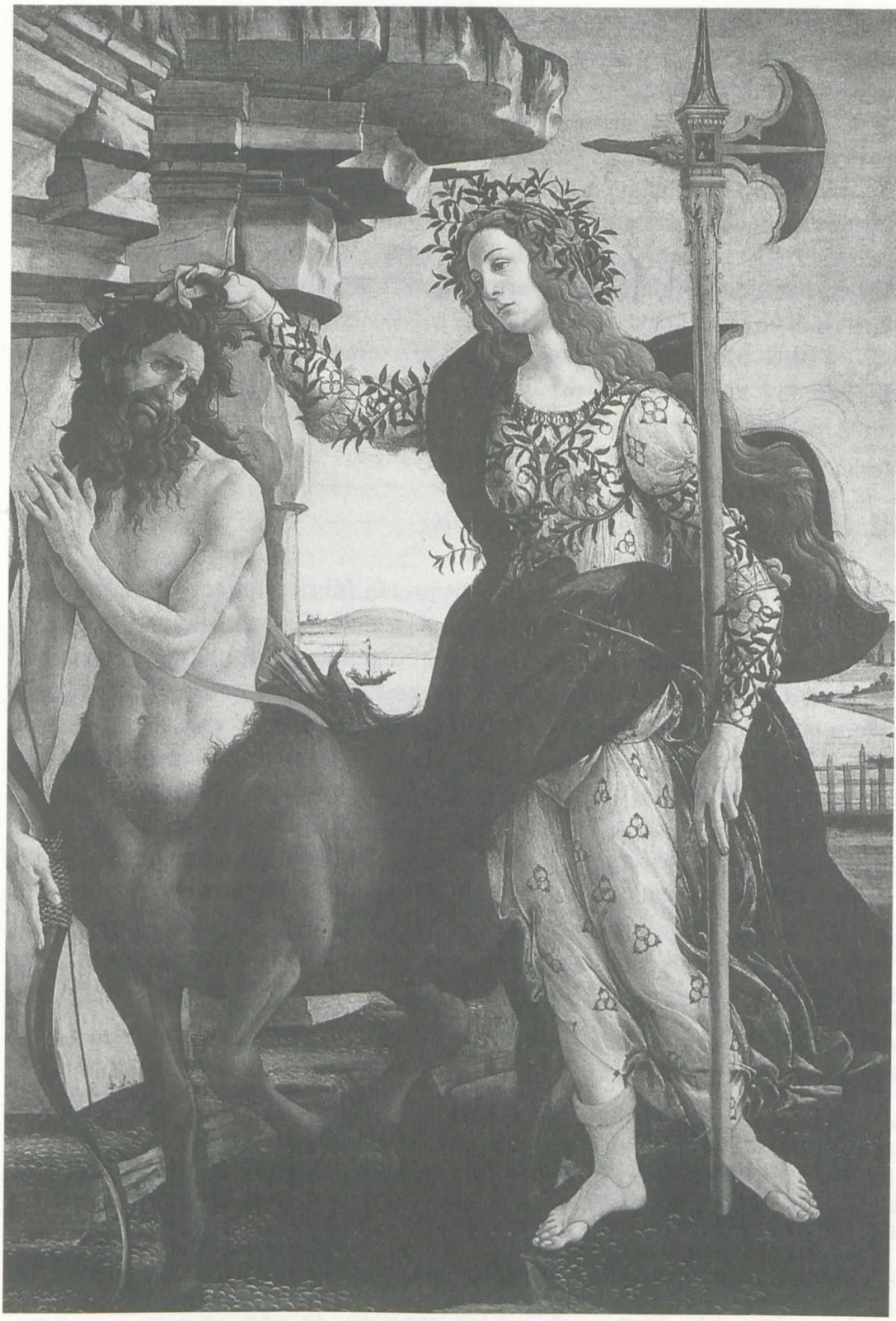

Abb. 3 Sandro Botticelli: Minerva und Kentaur. Tempera und Öl auf Leinwand $(207$ x $148 \mathrm{~cm})$. Florenz, Galleria degli Uffizi. 
weil mit dieser Kombination weder die Einzelfragen zum Bildprogramm hinreichend geklärt werden konnten, erst recht aber nicht der Sinn einer solchen Verknüpfung.

Im Folgenden soll gezeigt werden, daß wenige Verse aus einem im Auftraggeberumkreis bekannten spätantiken Text die inventio des Bildprogramms ausgelöst haben können, und daß diese Annahme um so wahrscheinlicher ist, als sich aus dem Kontext dieser Verse Hinweise auf den wahrscheinlichen Sinn der Bilderfindung ergeben. Ob die anschließenden Argumente ausreichen, einen Text als Quelle einer Bilderfindung zu etablieren, sei als grundsätzliches methodisches Problem hiermit exemplarisch zur Diskussion gestellt.

\section{De nuptiis Philologiae et Mercurii}

Die Erklärungsnot der Primavera-Interpreten führte vielfach dazu, daß man über die gern zitierte Literatur aus Antike und Neuzeit hinaus für Einzelfragen des Bildprogramms zusätzlich auf einen denkbar unklassischen spätantiken Lehrtraktat verwies. Gemeint sind die De nuptiis Philologiae et Mercurii libri des Martianus Capella, entstanden etwa zwischen 410 und 429. ${ }^{22}$ Martianus wurde zitiert, um den lange vermißten Beleg zu erbringen, daß der Gott Merkur in der Antike tatsächlich auch als Frühlingsgott (veris deus) aufgefaßt wurde. ${ }^{23} \mathrm{Da}$ das relativ keusche Auftreten der Venus in der Primavera nicht zu der weithin üblichen Vorstellung von der Laszivität dieser Göttin passen wollte, belegte man mit Hilfe von Martianus, daß Venus in der Antike nicht allein als Verkörperung der wollüstigen Liebe galt, ${ }^{24}$ sondern auch als Schutzgöttin der Ehe. ${ }^{25}$ Bis heute konnten unter den antiken Quellen allein bei Martianus Belege für diese Auffassungen gefunden werden.

22 Benutzte Ausgabe: Martianus Capella. Hg. von James Willis. Leipzig 1983 (Bibliotheca Scriptorum Graecorum et Romanorum Teubneriana). - Zur Textüberlieferung siehe Texts and Transmission. A Survey of the Latin Classics. Hg. von L. D. Reynolds. Oxford 1983, S. $245 f$.

Martianus Capella, S. 12; Dempsey: >Mercurius Ver<, S. 251-273.

24 So Lightbown: Botticelli, S. 127; Venus ist hier Beschützerin des Hymen, des römischen Gottes der Ehe, der mit den drei Grazien verwandt ist. In - wenn auch nicht direkter - Verbindung mit Hymen kann Venus in mythologisch-allegorischen Hochzeitszügen auftreten, so zum Beispiel in Vatikanstadt, cod. Urb. 899 (spätes 15. Jahrhundert; Le nozze di Costanzo Sforza e Camilla d'Aragona Imeneo; vgl. Mesura et arte del danzare. Guglielmo Ebreo da Pesaro e la Danza nelle corti italiane del XV secolo. Hg. von Patrizia Castelli, Maurizio Mingardi, Maurizio Padovan [Ausst.kat.]. Pesaro 1987, S. 24-25, Abb. 23 und 27). In diesem Zusammenhang wurde auch darauf hingewiesen, daß es bei Martianus heiße, die Erde feiere, indem sie sich mit leuchtenden Blumen bedecke, Merkurs Aufstieg zum Himmel, bei dem er erbitte, daß sein Vater Jupiter mit der Braut zu seiner Hochzeit herabsteigen möge: Lightbown: Botticelli, S. 135 (Martianus Capella, S. 20ff.). 
Die Bücher von der Hochzeit der Philologie und Merkurs waren als ganzes oder in Teilen seit dem 9. Jahrhundert eines der meist verbreiteten Schulbücher des Mittelalters und prägten entscheidend das Studium der sieben freien Künste. Ausgangspunkt des allegorisch verkleideten Traktats über die septem artes ist, daß Merkur sich entschließt zu heiraten. Apoll empfiehlt ihm die doctissima virgo Philologia, die daraufhin zur Göttin erhoben wird.

Die Vielzahl der Martianus-Handschriften ist bis heute nicht erfaßt, ${ }^{26}$ noch weniger die der zahlreichen Kommentare zu dem in ganz und gar unklassischem Latein verfaßten Text, der mehr als mancher andere nach Erläuterungen verlangt. Besonders der von Remigius von Auxerre (nach 841ca. 908) verfaßte Kommentar wurde fast ebenso bekannt wie die Hochzeit der Philologie selbst. ${ }^{27}$ In zahlreichen Handschriften wurde er parallel zum Text mitgeteilt; kaum ein zweiter Text ist so eng mit der Geschichte seiner Kommentierung verknüpft wie die Hochzeit der Philologie. ${ }^{28}$

Im Quattrocento, besonders in Florenz, erfuhr die Schrift - wenn auch unter veränderten Vorzeichen - eine neue Blüte. War sie im Mittelalter zumeist als Gebrauchstext überliefert worden, der selten und wenn doch, so meistens ohne größeren Aufwand illustriert wurde, ${ }^{29}$ so sind aus dem Quattrocento reich geschmückte Handschriften erhalten - auch solche aus Medici-Beständen. ${ }^{30} \mathrm{Im}$ Jahr 1441 gelangte eine bereits im 12. Jahrhundert entstandene illustrierte Martianus Capella-Handschrift in den Besitz der Medici. Es ist der heute in der Bibliotheca Laurenziana aufbewahrte Codex S. Marco 190. Dieser wurde von Angelo Poliziano benutzt, und es ist nicht unwahrscheinlich, daß auch Polizianos Schüler, Lorenzo di Pierfrancesco de' Medici, ihn zumindest kannte, wenn dieser nicht sogar mit Hilfe dieses Codex unterrichtet wurde. ${ }^{31}$ Zugleich diente diese Handschrift Martianus-

26 Claudio Leonardi: I codici di Marziano Capella. In: Aevum 33 (1959), S. 443-489; Aevum 34 (1960), S. 2-99.

27 Remigius Autissiodorensis: Commentum in Martianum Capellam. Hg. von Cora E. Lutz. Bd. 1-2. Leiden 1962-1965; vgl. auch Remigius Autissiodorensis: Commentum in Martianum Capellam. A Translation and Commentary by Philip M. Smith. PhD thesis Florida State University 1987. Zum Autor siehe C. Jeudy: Remigius von Auxerre. In: Lexikon des Mittelalters. Bd. 7. München 1995, S. 707f. (mit Lit.).

$28 \mathrm{Zu}$ den mittelalterlichen Martianus-Kommentatoren vgl. Jane Chance: Medieval Mythography. From Roman North Africa to the School of Chartres, A. D. 433-1177. Gainesville etc. 1994. Als einer der wichtigeren weiteren Kommentare sei der Bernardus Silvestris zugeschriebene genannt: The Commentary on Martianus Capella's De nuptiis Philologiae et Mercurii attributed to Bernardus Silvestris. Hg. von Haijo Jan Westra. Toronto 1986 (Studies and Texts 80).

29 E.-F. Corpet: Portraits des arts libéraux d'après les écrivains du moyen âge. In: Annales archéologiques 17 (1858), S. 89-103; Karl-August Wirth: Eine illustrierte Martianus CapellaHandschrift des 13. Jahrhunderts. In: Städel-Jahrbuch, Neue Folge 2 (1969), S. 43-74.

30 Leonardi: codici (1960), S. 36-48.

31 Leonardi: codici (1960), S. 47f. - Ludwig Heinrich Heydenreich: Eine illustrierte Martianus Capella-Handschrift des Mittelalters und ihre Kopien im Zeitalter des Frühhumanismus. In: Kunstgeschichtliche Studien für Hans Kauffmann. Berlin 1956, S. 59-66; Facsimile delle miniature di Attavante Fiorentino contenute nel codice Marciano Capella, Le nozze di Mercurio colla Filologia, che si conserva nella Biblioteca Marciana, fotografie eseguite da Antonio Perini. Venedig 1848. 
Illustratoren des Quattrocento als Vorlage. Als Beispiel dafür sei auf die wohl kurz vor Botticellis Primavera entstandene, illustrierte Martianus-Handschrift in Rom, Bibliotheca Vaticana, Cod. Urb. lat. 329, hingewiesen. ${ }^{32}$

\section{In quinto mense}

$\mathrm{Da}$ die Hochzeit der Philologie über diese Form der Überlieferung hinaus im 15. Jahrhundert ihre Blüten trieb, belegt ein in diesem Zusammenhang bisher unberücksichtigtes Bild: Es handelt sich um eine kolorierte Federzeichnung, die in einer in Frankreich (?) entstandenen Handschrift des frühen 15. Jahrhunderts eine anonyme lateinische Dichtung über das Wirken antiker Gottheiten im Frühlingsmonat Mai ergänzt (Abb. 4).

In quinto mense quo sol geminorum
Intrat amorose Veneris quo gratior ardor
Ignit amore suos tirones et vocat illos
Ad sua conubia varius quo satrapa Stilbon
Virginis ut capiat amplexus sepe regnat
Quem montana suo placat splendore Dyana
Quo stillata viget tellus ex floribus ortis
Quo spergit per humum sua pluralia florida Flora
Quae florum dea grata suis animantibus herbis
Precipit ut pratis honorent Phillida [Akk.!] veste
per quam prata vigent et florida iugera vernant
Su<c $\rangle$ crescunt segetes fructices lanugine condent
Arborei fructus redolent ex germine grato
Quo rosa candidior rubicundaque mirice tincta
Discerpunt cegetes [lies: segetes] capsarum roris ob auram
Vivifice lutis ut captant basia Phebi. ${ }^{33}$

Unter dem Sternzeichen der Zwillinge erscheint die Trias genau derjenigen Götter, deren Kombination für die Deutung der Primavera stets die größten Schwierigkeiten bereitet hatte: in der Mitte Venus, zu ihrer Rechten Merkur, der auf seinen Planeten weist, und zur Linken die Blüten streuende Flora - alle drei durch das zugehörige Gedicht eindeutig zu identifizieren.

Flora streut, dem Text zufolge, ihre Fülle über die Erde aus und befiehlt den Blumen, die Wiesen zu bekleiden. Indem sie so das Liebeslager bettet, wird sie zur Gehilfin der Venus - »ministra Veneris«, wie schon Remigius

32 L. H. Heydenreich. Die editio princeps des Textes erfolgte 1499.

33 Das Gedicht ist überschrieben mit »liber de regalia mundi vel de tribus statibus « (Cambridge, Trinity Hall, Ms. 12, [französische Übersetzung von Boethius, De consolatione philosophiae, mit genannter lateinischer Dichtung im Anhang], A. 15. Jh., fol. $100^{\mathrm{r}}-103^{\mathrm{r}}$, hier $\left.101^{\mathrm{vb}}\right)$ : Montague Rhodes James: A descriptive Catalogue of the Manuscripts in the Library of Trinity Hall. Cambridge 1907, S. 14-32, hier S. 29; Catalogue of astrological and mythological illuminated manuscripts of the latin middle ages. $\mathrm{Hg}$. von Harry Bober. III: Manuscripts in English Libraries, I, by Fritz Saxl and Hans Meier. London 1993, S. 432-437. 


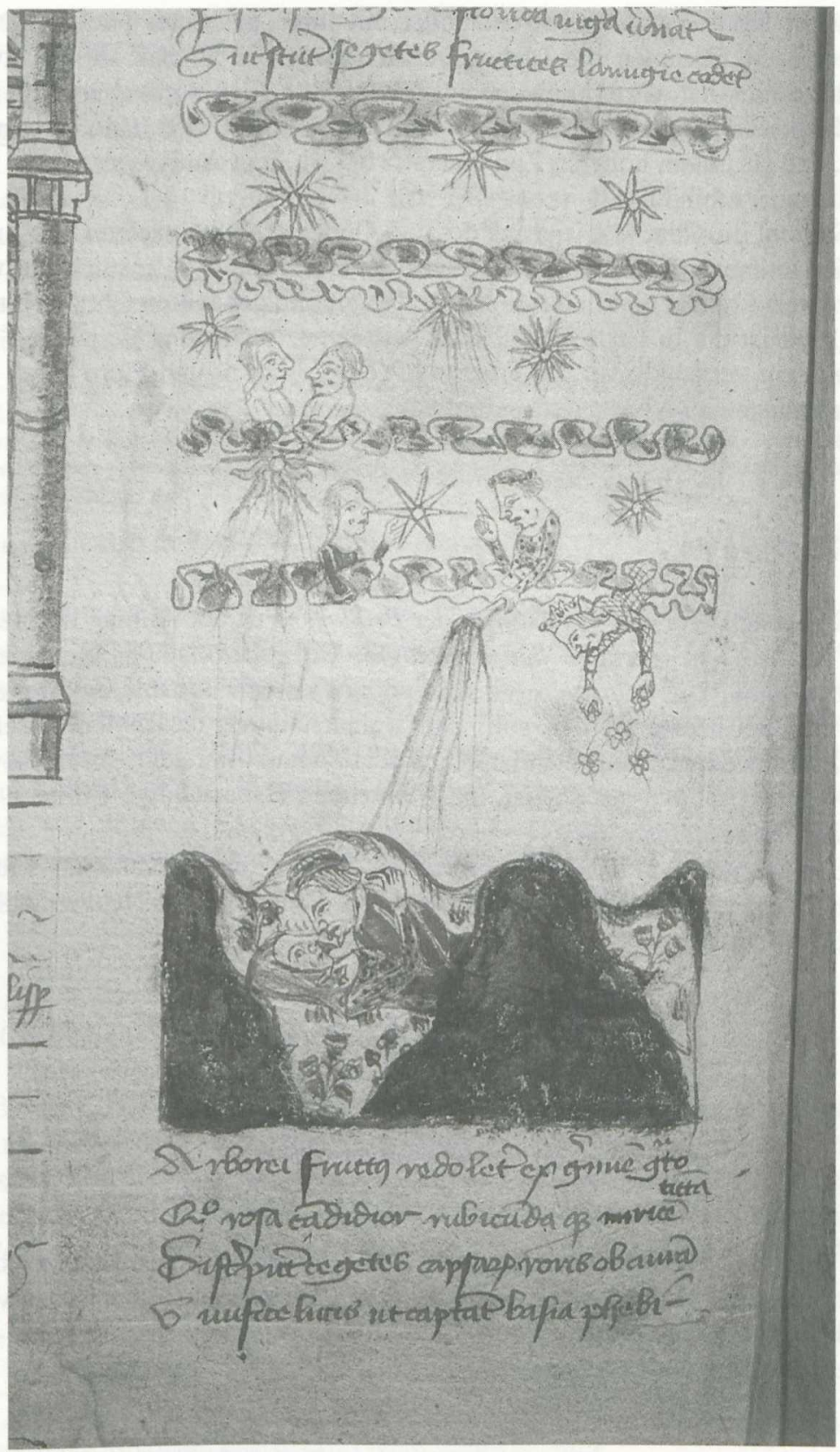

Abb. 4 Merkur, Venus und Flora unter dem Sternzeichen der Zwillinge.

Illustration zu einer anonymen lateinischen Dichtung über das Wirken antiker Gottheiten im Frühlingsmonat Mai. Cambridge, Trinity Hall, Ms. 12, fol. 101v. 
glossiert hatte. Venus selbst entzündet mit ihren aus einer Fackel herabgeworfenen Flammen die Liebe. Die Wirkung, die all das auf die zwei Erdbewohner hat, wird durch den auffälligen Hügel im Vordergrund ebenso verdeckt wie betont. Die Frivolität der Szene wird im Text dadurch gemildert, daß sie einem höheren Ziel unterworfen ist: dem Aufruf der Venus zur ehelichen Verbindung. ${ }^{34}$

Sowohl die Charakterisierung der drei Gottheiten im einzelnen, wie auch ihr Zusammenspiel setzen - wie auch immer vermittelt - letztlich die von Martianus Capella überlieferten Vorstellungen der genannten Götter voraus. Zugleich ist die in Gedicht und Bild vertretene Auffassung derjenigen der Primavera verwandt, wo die Trias von Venus, Merkur und Flora durch die Figurendisposition besonders betont ist.

\section{IV. hymenea}

Schlüsselstelle für das Verständnis der Primavera ist der Anfang des neunten Buches der Hochzeit der Philologie. Um Mißverständnissen gleich vorzubeugen: Die Primavera ist alles andere als eine Illustration zu dieser Textstelle. Gezeigt werden soll, daß wahrscheinlich die mythologischen Gestalten in der hier auftretenden Konstellation und Charakterisierung sowie der Kontext, in dem sie stehen, die weitgehend eigenständige Bildidee zur Primavera ausgelöst haben.

Das neunte Buch behandelt harmonia, die hier für die Kunst der Musik steht. Die personifizierte Harmonia ist es, die schließlich die Neuvermählten ins Schlafgemach geleitet.

DE HARMONIA
Iam facibus lassos spectans marcentibus ignes
instaurare iubet tunc hymenea Venus
quis modus inquit erit quonam sollertia fine
impedient thalamos ludere gymnasia
deriguit comis blandisque assueta Voluptas
et noster pallens contrahit ora puer
ipsa etiam fulcris redimicula nectere sueta
Flora decens trina anxia cum Charite est. ${ }^{35}$

Das Buch beginnt mit der Feststellung, daß das langwährende Hochzeitsfest Merkurs gerade - nach dem umfangreichen Programm, das mit der Vorstellung der vorausgehenden sechs freien Künste bereits absolviert ist - in einem Stadium der Ermattung angelangt ist:

34 "vocat illos ad sua conubia«. 
Als sie an den erschlafften Fackeln die Flammen nachlassen sah, rief Venus dazu auf, die »hymenaea zu erneuern.

Mit »hymenaea« dürfte in Verbindung zur Musik über das Hochzeitliche im Allgemeinen hinaus auch die Bedeutung von Hochzeitsliedern oder -tänzen mit angeklungen haben.

")Quis modus` inquit >erit?« « läßt Martianus dann Venus fragen und erinnert damit an den Hochzeitsaufruf in Vergils Aeneis, den dort Juno an die Liebesgöttin richtet. ${ }^{36}$ Während bei Vergil Venus die Eheschließung der Protagonisten strikt ablehnt, erweist sich die Göttin bei Martianus als Förderin der Ehe.

,Wie lange denn halten die Gymnasien, mit ihrer Klugheit am Ende, davon ab, das Ehelager zu bespielen? Voluptas, gewohnt freundlich zu sein und schmeichelnd, ist erstarrt, und unser erblaßter Knabe (i. e. Amor) hat das Gesicht zusammengezogen. Jene aber, gewohnt den Lagern Girlanden zu binden, die anmutige Flora, ist bei der dreifachen verängstigten Charis.

Venus ruft also ihr Gefolge, nämlich Amor, Flora und die drei Grazien dazu auf, das Fest zu erneuern: »Instaurare iubet tunc hymenaea Venus«. Die Folge dieses Aufrufs scheint in der Primavera ins Bild gesetzt, wo das gesamte Personal des zitierten Buchbeginns erscheint. Voluptas ist dabei, den üblichen Kommentaren entsprechend, nicht als eigene Gestalt sondern als eine Eigenschaft von Venus aufzufassen. ${ }^{37}$ Merkur wird zwar in diesem ersten Satz nicht erwähnt, seine Gegenwart ist jedoch vorausgesetzt. Gleich anschließend heißt es, Merkur sei bereits so von Begierde entflammt, daß er alles, was er nach Hochzeitsgebräuchen noch an Kostbarem vorbereitet habe, am liebsten fahren lassen wolle (ein Anblick, der Venus nicht mißfalle)..$^{38}$

\section{Chloris eram quae Flora vocor}

$\mathrm{Da}$ von Chloris und Zephyr im Text nicht die Rede ist, scheint dazu zu zwingen, wieder bei den oben kritisierten Kunstgriffen Zuflucht nehmen zu müssen. Die hier vertretene These allerdings ist, daß die genannte Textpassage in der Form, wie sie wahrscheinlich damals rezipiert wurde, den Grundgedanken für das Bild Botticellis anregen konnte, das heißt unter Einbindung

36 »Sed quis erit modus, aut quo nunc certamine tanto? Quin potius pacem aeternam pactosque hymenaeos exercemus?«(Vergil: Aeneis IV, 98f.).

37 „VENUS id est voluptuosa. VENUS mater Hymenei« (Remigius: Commentum. Hg. von C. Lutz. Bd. 2, S. 294; Remigius: Commentum. Hg. von P. Smith, S. 100-101).

38 »... quin etiam ipsum Atlantiadem tam flammatae cupiditatis cura concussit, ut omittere vellet, quae circa sponsalem conventum ornatiora disposuit: tanti quippe visum numquam Veneri displicere« (Martianus Capella, S. 338). 


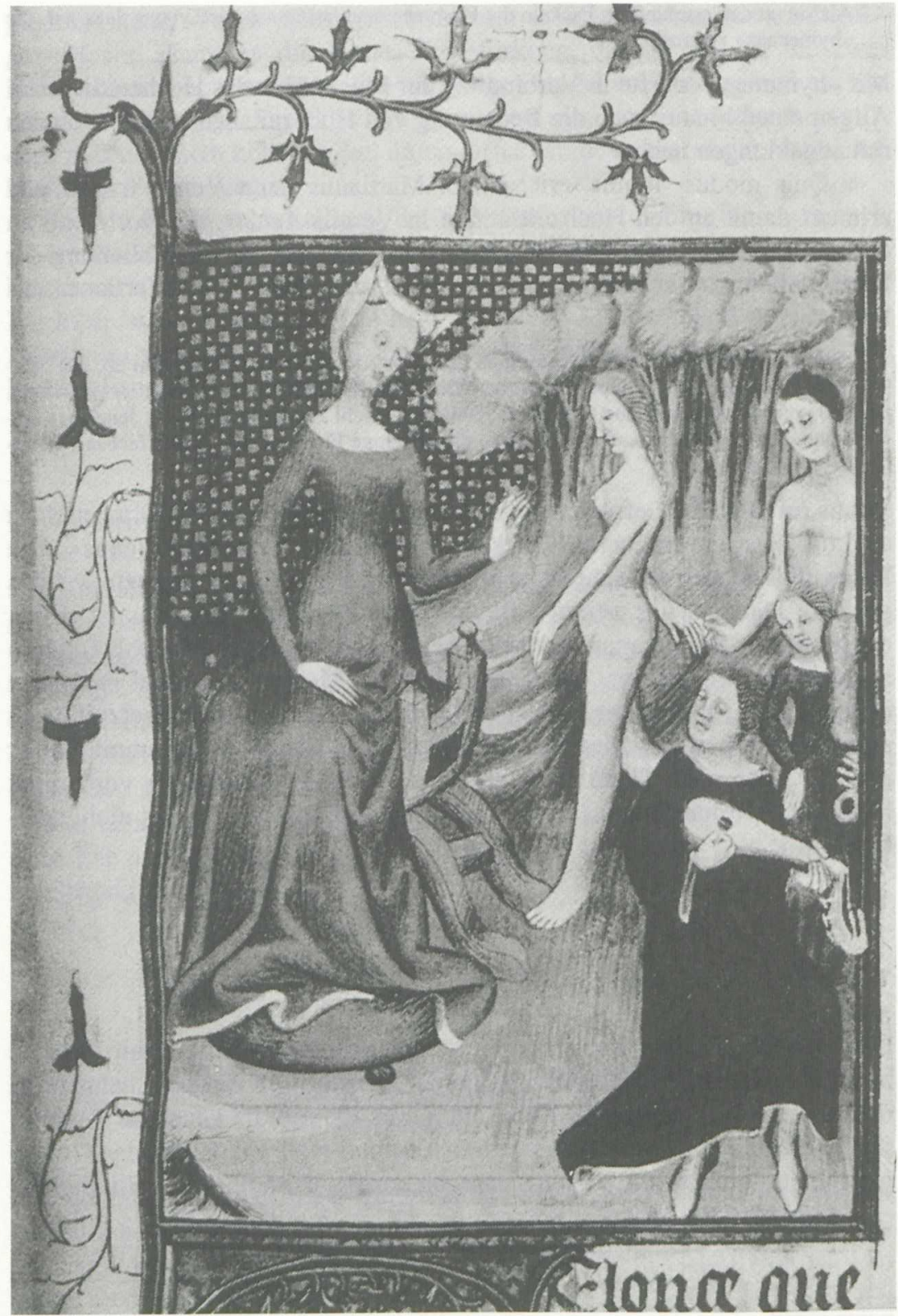

Abb. 5 Flora mit einem Liebespaar. Illustration zu Giovanni Boccaccio: De claris mulieribus. London, British Library, Ms. Royal 20 C V, fol. 101r. 


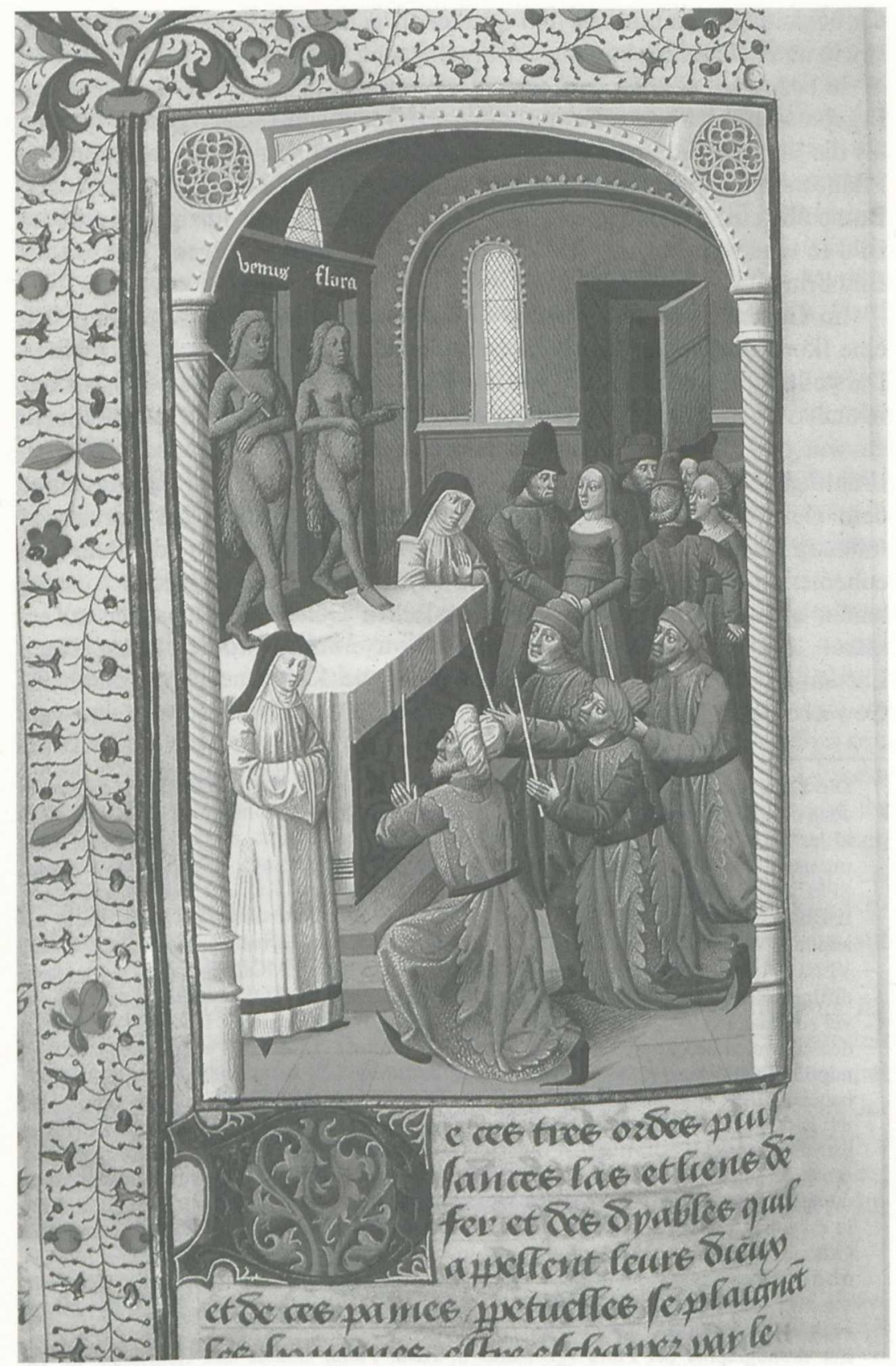

Abb. 6 Von Heiden verehrte Idole der Venus und Flora. Illustration zu Augustinus: De civitate dei. Den Haag, Rijksmuseum Meermanno-Westreenianum, Ms. 11, fol. 89v. 
der bekannten Kommentare und der damals geläufigen literarischen Bildung sowie unter Berücksichtigung der bestehenden Bildtradition. ${ }^{39}$

In bezug auf Chloris und Zephyr ist zunächst festzuhalten, daß sie - den Ergebnissen der letzten Restaurierung zufolge - wohl später gemalt wurden als die übrigen Figuren. Als einzige sind sie über den bereits ausgeführten Pflanzenhintergrund gemalt. ${ }^{40}$ Sie nahmen also schon im Arbeitsprozeß Botticellis eine Sonderstellung ein. Zugleich sind sie als einzige Figuren im Bild in eine sehr bewegte Handlung verstrickt, die eine eigene historia ins Bild bringt.

Im Gegensatz zu den übrigen Göttergestalten der Primavera hatte sich eine Ikonographie der Göttin Flora im Quattrocento noch nicht ausgeprägt. Darstellungen von Flora, die vor der Primavera entstanden, sind bisher nur in außeritalienischen Beispielen bekannt (vgl. Abb. 4-6). ${ }^{41}$ So war Botticelli gezwungen, die Gestalt der bis dahin in Italien nur literarisch fixierten Frühlingsgöttin in besonderer Weise kenntlich zu machen. Remigius hatte dem Namen der Göttin Flora lediglich die Erläuterung »dea florum vel ministra Veneris « hinzugefügt. Bekannt war im Quattrocento vor allem eine euhemeristische Erklärung des Flora-Mythos. ${ }^{42}$ Frühe christliche Autoren hatten nicht selten versucht, die heidnischen Götter für Menschen zu erklären, die erst im Nachhinein zu Göttern erhoben worden seien. Nach Lactantius (fl. Anfang 4. Jahrhundert) hat die Kurtisane Flora, durch ihr Gewerbe reich geworden, das Volk von Rom als Erbe bestimmt und ver-

39 Die Kommentierung der genannten Stelle durch Remigius lautet: »MUSICA dicitur ab aqua eo quod in lymphis, id est in undis, reperta est prius ab hominibus. IAM FACIBUS id est faculis vel flammis, MARCENTIBUS id est sopitis et deficientibus, nocte imminente. LASSOS id est deficientes, sole vergente ad occasum, IGNES id est lucernas, SPECTANS scilicet ipse Iuppiter, id est considerans. INSTAURARE id est emendare, IUBET scilicet Iuppiter vel Pallas, Apollinem vel aliam dotalium. HYMENEIA id est nuptialis vel voluptuaria. HYMENEA pro Hymeneia, quia Hymeneus filius Veneris fuit. VENUS id est voluptuosa. VENUS mater Hymenei. QUIS MODUS scilicet narrandi et diffiniendi, ERIT? QUONAM pro quo, FINE id est termino. SOLLERTIA id est studiosa vel sollicita. GYMNASIA exercitia. THALAMOS nuptias geniales. DIRGUIT obstupuit, defecit, vel induruit. COMIS ornata, pulchra, facetosa. BLANDIS scilicet rebus, id est nuptiis, QUE vacat. CONTRAHIT torpet, constringit, et contristatur, vel rugosa ORA habet, PUER id est Cupido filius meus. ISPA ETIAM FLORA id est dea florum vel ministra Veneris, cuius festa Kalendis Aprilibus colebantur, id est Floralia. FULCRIS id est toris, lectis. REDIMICULA ornamenta. REDIMICULA TRINA id est coronulas vel ornacula, id est tria fila varii coloris, id est rubri, purpurei, iacinctini, quibus capita nuptarum in modum coronae nectebantur. NECTERE iungere, SUETA scilicet ipsa Flora, id est solita. DECENS pulchra, honesta, CUM TRINA CARITATE EST id est cum tribus Gratiis. Tres sunt Caritae sorores quae conciliant nuptias et omnem amorem. ANXIA tristis. « (Remigius: Commentum. Hg. von C. Lutz. Bd. 2, S. 294; Remigius: Commentum. Hg. von P. Smith, S. 99-103).

40 Lightbown: Botticelli, S. 145.

41 Zur Ikonographie der Flora vgl. Julius S. Held: Flora, Godess and Courtesan. In: De artibus opuscula XL. Essays in honor of Erwin Panofsky. Hg. von Millard Meiss. New York 1961. Bd. 1, S. 201-218, Bd. 2, S. 69-74.

42

Lactantius: Divinarum institutionum libri. In: Corpus scriptorum ecclesiasticorum Latinorum. Bd. 19. Wien 1890, lib. I, 20, S. 72f.; vgl. auch: Scholia in Iuvenalem vetustiora. Hg. von Paulus Wessner. Leipzig 1931, S. 89f. 
fügt, daß mit dem Ertrag jährlich an ihrem Geburtstag ihrer durch Spiele gedacht werde. Der Senat habe den Spielen einen anderen Sinn geben wollen und eine Göttin der Pflanzenblüte mit dem Namen Flora erfunden, deren Mythos Ovid geschildert habe. Diese Erklärung war im Italien des Quattrocento vor allem durch Giovanni Boccaccio verbreitet, denn die Rückführung der in der Antike gefeierten Flora auf eine Menschengestalt, hatte es Boccaccio erlaubt, Flora in de mulieribus claris in die Lebensschilderungen berühmter Frauen einzureihen ${ }^{43}$ Allerdings schilderte Boccaccio nicht allein das Leben der angeblichen Kurtisane, sondern auch den Mythos der Frühlingsgöttin, der unter den antiken Quellen allein in Ovids Fasti ausführlicher mitgeteilt wird.

Dort heißt es, ihr ursprünglicher, griechischer Name sei Chloris gewesen. Diesen gebe man im Lateinischen mit Flora wieder. Sie sei eine Nymphe in den Gefilden der Seligen gewesen, wo im Frühling Zephyr sie gesehen, verfolgt und überwältigt habe. Dann habe er sie zur Frau genommen und ihr als Morgengabe Land geschenkt. In dessen Mitte liege ein fruchtbarer Garten mit einer Wasserquelle, den Zephyr mit edlen Blumen gefüllt und über den er sie eingesetzt habe. ${ }^{44}$

Von den französischen Boccaccio-Illustrationen des 15. Jahrhunderts zeigt keine die so von Ovid geschilderte Frühlingsgöttin. Zumeist wurde Flora als Regentin über Liebespaare dargestellt. Das in der British Library in London aufbewahrte Royal Ms. $20 \mathrm{CV}$ zum Beispiel zeigt, wie ein unbekleidetes Liebespaar vor die auf einem Faldistorium sitzende Flora tritt (Abb. 5). Gelegentlich zeigte man auch die Kurtisane Flora mit einem reichen Verehrer (mit auffälligem Geldbeutel), im Hintergrund die von ihr gestifteten Floralia, bei denen nackte Tänzerinnen vor dem auf Tribünen sitzenden Publikum auftreten. ${ }^{45}$ Eine Civitas dei-Handschrift von 1478 veranschaulicht jenen heidnischen Götzendienst, den der Kirchenvater Augustinus verurteilte: Die Miniatur auf fol. $89 \mathrm{v}$ schildert, wie Römer die auf einem Altar stehenden Idole von Venus und Flora verehren (Abb. 6).

Botticellis Primavera ist somit das früheste bekannte Bildzeugnis für den Flora-Mythos nach Ovid, der, wie erwähnt, durch Boccaccio vermittelt sein konnte. Wollte man die Gestalt der Göttin Flora positiv gedeutet und nicht mit einer bloßen Frühlingshore verwechselt wissen, wie sie in der Geburt der Venus erscheint, war man - bei Verzicht auf erläuternde Beischriften - geradezu gezwungen, Motive aus Ovids Fasti aufzugreifen, denn diese allein bieten unter den antiken Quellen genügend Hinweise für eine Ikonographie der Frühlingsgöttin.

43 De mulieribus claris, cap. LXIV: Giovanni Boccaccio: Tutte le opere. Hg. von Vittore Branca. Bd. 10. Mailand ${ }^{2} 1970$, S. 256-261; vgl. Giovanni Boccaccio: Genealogie deorum gentilium libri. Hg. von Vincenzo Romano. Bd. 1. Bari 1951 (Scrittori d'Italia 200), S. 218.

44 Ovid: Fasti. Hg. von Franz Bömer. Bd. 1. Heidelberg 1957, S. 230-240.

45 Flora und ihr Geliebter Fanutius. Illustration zu Giovanni Boccaccio, De claris mulieribus. New York, Public Library, Spencer Collection, ms. 33, fol. 35v, - Vgl. Held: Flora, Abb. 12. 
Man muß demnach die Dreiergruppe von Flora, Chloris und Zephyr, aus der sich die Gestalt der Flora isoliert (vgl. Abb. 1), als eine die mythische Metamorphose zur Göttin einbeziehende Darstellung der von Martianus genannten Flora verstehen, Chloris und Zephyr also zunächst als eine Art Bildglosse zur Gestalt der Göttin auffassen.

Mit dieser positiv geschilderten Göttin Flora ist die mit ihr untrennbar verknüpfte Vorstellung von Frühling und vielleicht auch die von Frühlingsfest im Bild repräsentiert. Remigius hatte darauf hingewiesen, daß zu Ehren der Flora in der Antike im Frühjahr die Floralia gefeiert wurden. Für die Zeitgenossen Botticellis mag der Gedanke des Frühlingsfests durch die vorgestellten Kostüme hervorgerufen worden sein. Des öfteren ist darauf hingewiesen worden, daß Parallelen zur Festkostümierung des Quattrocento bestehen, insbesondere zu jener der in der Florentiner Kultur fest verankerten Calendimaggio-Feiern. Die Tage dieses Frühlingsfests überschneiden sich mit jenen der antiken Floralia. So ist nicht auszuschließen, daß man im Quattrocento mit den Calendimaggio-Feiern eine Art Wiederbelebung der antiken Festkultur verband. Schließlich boten die Floralia schon aufgrund der Etymologie des Namens Florenz eine willkommene Anknüpfung. ${ }^{46}$

\section{VI. tria fila varii coloris}

Über die Charakterisierung der Einzelfiguren und deren Konstellation hinaus deutet die formale Gestaltung der Primavera darauf hin, daß bestimmte Konzepte, die sich aus dem Kontext der genannten Martianus-Verse ergeben, für das Programm des Bildes von Bedeutung waren.

Selbst ein weithin bekanntes Mißverständnis der Hochzeit der Philologie blieb in diesem Zusammenhang offenbar nicht ohne Wirkung auf die Primavera. Das trina in Martians Text bezieht sich sicher auf Charis, um die Dreizahl der hier im Singular genannten Chariten oder Grazien hervorzuheben. Remigius aber bezog trina zugleich auf redimicula, den Blumenschmuck also, den Flora für das Hochzeitslager bereitet. ${ }^{47}$ Diese redimicula setzen sich - so Remigius - aus drei Strängen zusammen, die drei verschiedene Farben besitzen. Und er kann diese Farben sogar benennen: ruber, purpureus und iacinctinus - rot, purpur- und hyazinthfarben. Es fällt auf, daß Botticelli in der Primavera mit einer relativ reduzierten Farbskala operiert. Vor dem in dunkles Grün getauchten Hintergrund herrschen drei Farben vor: Die Blüten im Bausch der Flora sind weiß, rot und blau; und auch das übrige Bild ist in unterschiedlichen Abstufungen von diesen drei

46 Vgl. jüngst Paola Ventrone: Lorenzo's Politica festiva. In: Lorenzo The Magnificent. Culture and Politics. Hg. von Michael Mallett und Nicholas Mann. London 1996 (Warburg Institute Colloquia III), S. 105-116.

47

»REDIMICULA TRINA id est coronulas vel ornacula, id est tria fila varii coloris, id est rubri, purpurei, iacinctini, quibus capita nuptarum in modum coronae nectebantur«. 
Farben dominiert, besonders in der Kleidung der Figuren. Während in den Blüten die drei Farben ganz blaß erscheinen und jeweils eine Spur der Farben der Nachbarblüten widerspiegeln, sind sie in den Kleidern der Figuren kräftiger ausgeprägt. Allein bei Venus, im Zentrum des Bildes, kommen Weiß, Rot und Blau gemeinsam in relativ ausgewogenem Verhältnis vor. Die übrigen weiblichen Figuren tragen überwiegend ein durchsichtiges Weiß, Merkur Rot, Zephyr Blau. Offenbar wollte Botticelli damit auf den mythischen Ursprung des Braut- und Brautlagerschmucks in den dreierlei Blüten der Flora hinweisen.

Die Dreizahl der Farben entspricht einer mehrfachen Dreigliederung in der Figurenanordnung: Die Aufteilung des Bildes in die zentrale Gestalt der Venus mit Amor und die zur Rechten und Linken der Liebesgöttin angeordneten Figuren wird auch durch die Disposition der Pflanzen im Hintergrund unterstrichen. Außerdem beherrschen drei mal drei Gestalten das Bild: Die Grazien bilden eine Dreiergruppe und auch Flora schließt sich mit Chloris und Zephyr zu einer solchen zusammen. Übrig bleiben die drei einzeln erscheinenden Merkur, Amor und Venus. Diese mögen durch ihre mythische Verbindung - Amor gilt als Frucht der Vereinigung von Venus und Merkur - ebenso als Dreiheit angesehen worden sein..$^{48}$ Nimmt man zudem den von Vasari überlieferten Titel Primavera, der sich aus drei mal drei Buchstaben zusammensetzt, als ursprünglich an, so verknüpfen die mittleren drei Buchstaben MAV (priMAVera) die Initialen der drei einzeln auftretenden Götter Merkur, Amor und Venus - zugegeben: ein gewagtes Gedankenspiel, das jedoch vor dem Hintergrund ähnlicher Buchstaben-, Wort-, und Zahlenspiele in der Literatur des Cinquecento nicht ganz absurd erscheinen mag. ${ }^{49}$

Schließlich kann man auch die drei relativ isoliert auftretenden Gottheiten Merkur, Venus und Flora als eine das Bild übergreifende Trias auffassen, wie sie auch in dem bereits gezeigten Mai-Bild vorkommt (Abb. 4).

Das bewußte Spiel mit der Dreizahl mag ein weiterer Grund dafür gewesen sein, die sieben bei Martianus genannten Gestalten zu Beginn des 9. Buchs um Chloris und Zephyr zu ergänzen.

\section{VII. harmonia}

Der in Farbigkeit und Bilddisposition erkennbare Dreierrhythmus kann in mehrfacher Hinsicht auf das im 9. Buch der Hochzeit der Philologie angeschlagene Leitmotiv der harmonia bezogen werden: Er läßt sich auf die Musik und deren Theorie ebenso beziehen wie auf jene der bildenden Kunst.

48 Lightbown: Botticelli, S. 135.

49 Freundlicher Hinweis: Friedrich Niewöhner, Wolfenbüttel. 
Bereits die Neunzahl ist eng mit der musikalischen harmonia verbunden, und es ist wohl schon bei Martianus kein Zufall, daß sie ausgerechnet in Buch 9 behandelt wird: Schließlich galten die neun Musen nach antiker Überlieferung als Töchter der Harmonia. Nach im Florenz des Quattrocento geläufiger Vorstellung entstehen durch die Umdrehung der Himmelssphären neun Klänge, die eine consonantia bilden und die wegen ihres Zusammenklingens auch die neun Musen genannt werden. ${ }^{50}$ Laut Marsilio Ficino entspricht diese consonantia der celestis harmonia, der himmlischen Harmonie. ${ }^{51}$

Die oft bemerkten Tanzschritte des Bildpersonals der Primavera (vgl. Abb. 1), insbesondere der drei Grazien, deuten darauf hin, daß sich die Aufforderung der Venus im Beginn eines Tanzes äußert. ${ }^{52}$ Für Zeitgenossen mag etwa der Gedanke an das in Florenz seit dem 13. Jahrhundert geläufige Tanzlied im Dreierrhythmus, die Ballata, nahegelegen haben, zumal das von Botticelli angeschlagene Frühlingsthema für die Ballata durchaus typisch ist. Als Beispiel sei auf Francesco Landinis Ecco la primavera hingewiesen:

Ecco la primavera
Che'l cor fa rallegrare,
Temp' è d'annamorare
E star con lieta cera.
No' vegiam l'aria e'l tempo
Che pur chiam'allegrezza.
In questo vago tempo
Ogni cosa ha vaghezza.
L'erbe con gran freschezza
E fior' copron i prati
E gli alberi adornati
Sono in simil manera.

$50 \mathrm{Zu}$ den verschiedenen theoretischen Konzepten zur Musik in der Renaissance vgl. Willem Elders: Symbolic scores. Studies in the Music of the Renaissance. Leiden, New York und Köln 1994 (Symbola et Emblemata. Studies in Renaissance and Baroque Symbolism). Klaus Wolfgang Niemöller: Zum Paradigmenwechsel in der Musik der Renaissance. Vom »numerus sonorus « zur »musica poetica«. In: Literatur, Musik und Kunst im Übergang vom Mittelalter zur Neuzeit. Bericht über Kolloquien der Kommission zur Erforschung der Kultur des Spätmittelalters 1989-1992. Hg. von Hartmut Boockmann u. a. Göttingen 1995 (Abhandlungen der Akademie der Wissenschaften in Göttingen, phil.-hist. Klasse, 3. Folge, Nr. 208), S. 187-215 (mit weiterer Literatur).

51 Marsilio Ficino: Commentarium in convivium Platonis, de amore. Über die Liebe oder Platons Gastmahl. Übersetzt von Karl Paul Hesse. Herausgegeben und eingeleitet von Paul Richard Blum. Lateinisch-Deutsch. Hamburg ${ }^{2} 1984$, Oratio quinta, cap. XIII, S. 180: »Preterea velocissima illa et ordinatissima celorum conversione musicam nasci consonantiam arbitramur atque octo circulorum motibus tonos octo, ex cunctis autem nonum quemdam produci concentum. Novem itaque celorum sonos a musica concordia musas novem cognominamus. Huius musice ratione noster olim donatus est animus. Cui enim est origo celestis, merito celestis innata dicitur harmonia«. - Vgl. dazu Elders: Symbolic scores, S. 211-251. $\mathrm{Zu}$ diesem Aspekt vgl. Patrizia Castelli: Il moto aristotelico e la slicita scientiar. Guglielmo Ebreo e la speculazione sulla danza nel XV secolo. In: Mesura et arte del danzare (wie Anm. 24), S. 34-57.

53 Hier zitiert nach: Das Cambridge Buch der Musik. Hg. von Stanley Sadie und Alison Latham. Aus dem Englischen von Dagmar Kreye und Christian Spiel. Frankfurt a. M. ${ }^{7} 1995$. 
Auch diese Liedform ist über den Rhythmus hinaus durch weitere Dreigliederung gekennzeichnet: Sie hat drei Strophen, deren erste am Schluß wiederholt wird.

Der auffällige, wohl bewußt vieldeutige Gestus der rechten Hand der Venus in Botticellis Gemälde ist in diesem Zusammenhang als Taktangeben oder Dirigieren zu verstehen. ${ }^{54}$ Die etwa auf Brusthöhe erhobene Hand mit leicht gekrümmten, zumeist nicht ganz geschlossenen Fingern läßt sich in bildlichen Darstellungen musikalischer Aufführungen häufig in diesem Sinne wiederfinden, sei es bei den musizierenden Engeln im Genter Altarretabel van Eycks (Abb. 7), ${ }^{55}$ sei es - in der direkten Umgebung Botticellis - im Relief singender Knaben von Luca della Robbia an der Sängerkanzel des Florentiner Doms. ${ }^{56}$

$\mathrm{Da} \beta$ keine Musikinstrumente sichtbar und daß es Götter sind, die die musikalische harmonia heraufbeschwören, deutet allerdings darauf hin, daß Botticelli im Gemälde weniger auf die irdische, für das Ohr hörbare Musik hinweisen wollte. Diese ist ohnehin in der Hochzeit der Philologie ebenso wie in der Musiktheorie des Quattrocento nur ein Aspekt von harmonia. Als Pendant höherer Rangordnung erörtert Martianus Capella die himmlische, für das menschliche Ohr nicht hörbare Musik. Und auch für Marsilio Ficino ist die mit Instrumenten oder Gesang ausgeübte Musik lediglich eine Imitation der in der menschlichen Seele eingeborenen himmlischen Harmonie. ${ }^{57}$ Von diesem himmlischen Urbild, nicht von der irdischen Musik wollte offensichtlich Botticelli mit Hilfe von Gestalten der antiken Mythologie ein Bild schaffen, oder zumindest die Regeln jener Harmonie für die bildende Kunst fruchtbar machen..$^{58}$

Die Vorstellung himmlischer Musik war der bildenden Kunst nicht ganz fremd: Die glücklich im Himmelreich angelangten Seelen werden in Bildern nicht selten von musizierenden Engeln begleitet (in der Regel allerdings ohne daß man dabei auf die Darstellung von Musikinstrumenten verzichtete). Mit dem sogenannten himmlischen Reigen in einem Vorgarten zum himmlischen Paradies hatte zum Beispiel Fra Angelico in seiner Gerichtsdarstellung in San Marco in Florenz eine Art christliches, allerdings endzeitliches Gegenstück für die Allegorie Botticellis geliefert. ${ }^{59}$

54 So hatte ihn bereits Adolfo Venturi in seinem in Rom 1925 erschienen Buch zu Sandro Botticelli interpretiert. Zu der Fülle weiterer Deutungen des Gestus vgl. Gombrich (wie Anm. 5), S. 11-12, Anm. 4. Vgl. dazu Elders: Symbolic scores, S. 216-220.

56 John Pope-Hennessy: Luca della Robbia. Oxford 1980, Abb. 12.

57 Ficino: Commentarium, Oratio quinta, cap. XIII, S. 180: »Huius musice ratione noster olim donatus est animus. Cui enim est origo celestis, merito celestis innata dicitur harmonia. Quam deinde variis instrumentis et cantibus imitatur.« Vgl. die sich an der zeitgenössischen Musiktheorie orientierende Deutung bei Wind: Pagan mysteries.

59 Vgl. Luciano Berti, Bianca Bellardoni und Eugenio Battisti: Angelico a San Marco. Rom 1965, Fig. 5-14. 


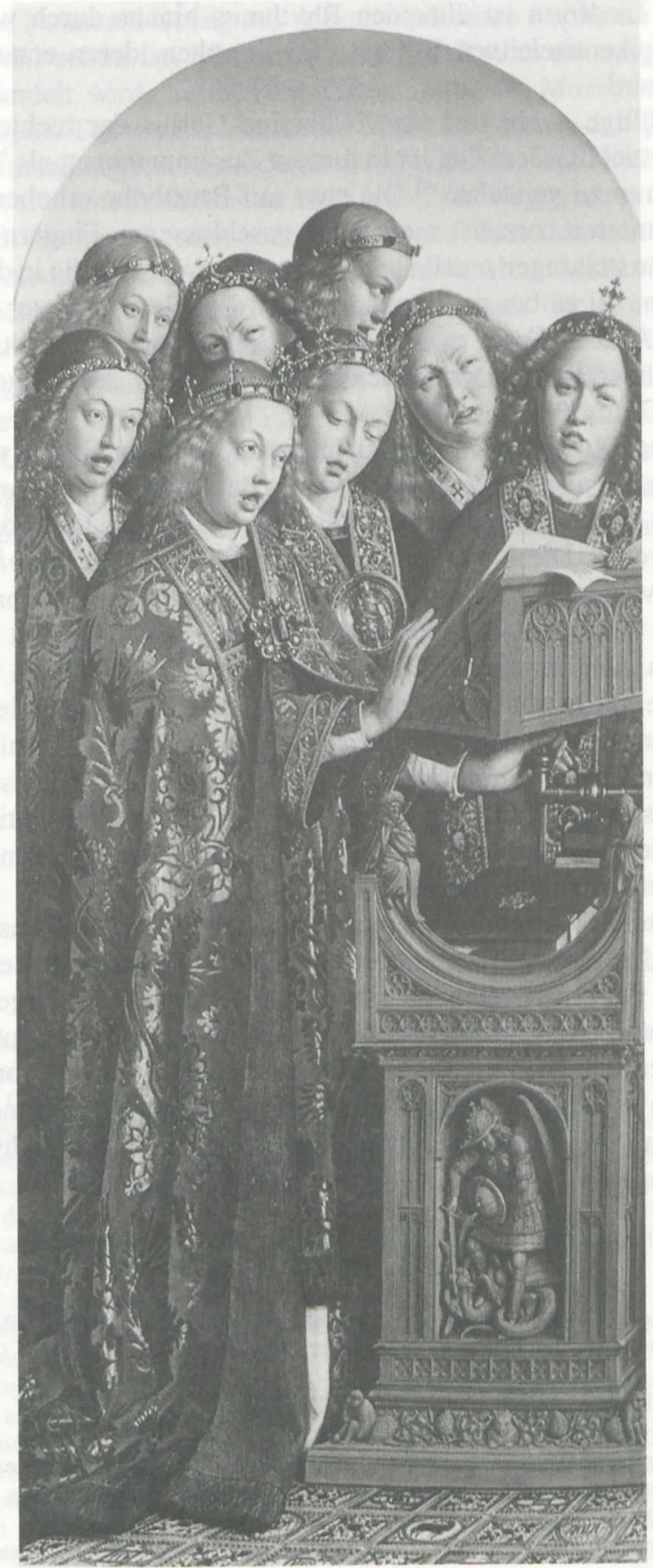

Abb. 7 Jan van Eyck: Musizierende Engel. Ausschnitt (um 1430) aus dem Altarretabel in Gent, St. Bavo. 
Über die Anspielungen auf die Musik hinaus fällt auf, daß die in der Primavera verwirklichten ästhetischen Ideale eng mit den von Leon Battista Alberti erstellten kunsttheoretischen Grundsätzen übereinstimmen, worauf - zumeist ohne nähere Begründung - bereits desöfteren hingewiesen wurde. ${ }^{60}$ Harmonia ist bei Alberti im Bereich von Malerei und Architektur durch concinnitas begründet oder mit dieser synonym. Die harmonische Zusammensetzung gilt Alberti als Quelle für die ästhetischen Ideale gratia und decor. Besonders die mit der Dreizahl spielende, komplexe Figurendisposition der Primavera deutet auf einen Bezug zu der von Alberti vertretenen Gesetzmäßigkeit hin, nach der es drei Dinge sind, die concinnitas begründen: numerus, finitio und collocatio.$^{61}$ Numerus (Zahl) und collocatio (Anordnung) sind langbewährte Konzepte der Ästhetik, besonders im Bereich der Musiktheorie. ${ }^{62}$ Finitio ist ein relativ schillernder Begriff, der hier wohl im Sinne von Regelhaftigkeit oder Gesetzmäßigkeit zu verstehen ist. Alle drei Kriterien haben offensichtlich für die Komposition der Primavera eine wichtige Rolle gespielt. Die aus diesen Prinzipien erwachsenden Ideale grazia und decor sind nicht allein formal in der Primavera verwirklicht, sondern auch in übertragener Gestalt im Bild präsent: Die drei Grazien zumindest stehen sicher - wie in der Renaissanceliteratur häufig als Göttinnen der Anmut zugleich für den Begriff der grazia. ${ }^{63} \mathrm{Ob}$ die blumenstreuende Flora, als diejenige, die die Wiesen schmückt, decor repräsentiert, muß mangels weiterer Belege zunächst Hypothese bleiben.

All das deutet darauf hin, daß die Primavera über die mythisch-allegorischen Einzelgestalten hinaus als ganze auch im Sinne einer Allegorie der Harmonie verstanden worden sein kann. Und auch als solche kann sie wenn man die Kenntnis Martianus Capellas voraussetzt - von den Zeitgenossen als Hinweis auf die Ehe verstanden worden sein. Denn derjenige, der in der Hochzeit der Philologie das universelle Prinzip der Harmonie vertritt, ist der Ehegott Hymen, da dieser Verschiedenes in Einklang zu bringen vermag. ${ }^{64}$

60 So von Dempsey: Portrayal, S. 165-166.

61 Leon Battista Alberti: L'Architectura (De re aedificatoria). Hg. von Giovanni Orlandi. Bd. 2. Mailand 1966, S. 815: „Ex quo statuisse possumus [...] praecipua esse tria haec, in quibus omnis, quam quaerimus, ratio consumetur: numerus, et quam nos finitionem nuncupabimus, et collocatio. Sed est amplius quippiam ex his omnibus compactis atque nexis, quo tota pulchritudinis facies mirifice collucescat: id apud nos concinnitas nuncupabitur, quam eandem profecto omnis esse gratiae atque decoris alumnam dicimus.« (ed. princ. 1485 mit Dedikation an Lorenzo de'Medici, verfaßt von Poliziano).

62 Bereits Augustinus hatte die Schönheit der Musik auf »aequalitas numerosa (zahlenmäßige Übereinstimmung) und »ordo« (Anordnung) zurückgeführt: Aurelius Augustinus: De musica libri VI. Hg. von Jacques Paul Migne. In: Patrologiae ..., series Latina. Bd. 32. Paris 1841, Sp. 1079-1194, hier cap. XIII, Sp. 1184; vgl. auch Augustinus: De civitate dei libri. In: Corpus Christianorum. Series Latina. Bd. 48. Turnhout 1955, lib. XXII, 19, S. 838. Siehe auch Werner Beierwaltes: Aequalitas numerosa. Zu Augustins Begriff des Schönen. In: Wissenschaft und Weisheit 38 (1975), S. 140-157; K. W. Niemöller (wie Anm. 50).

63 Mertens: Grazien, S. 179ff.

64 Chance: Mythography, S. 260-262. 


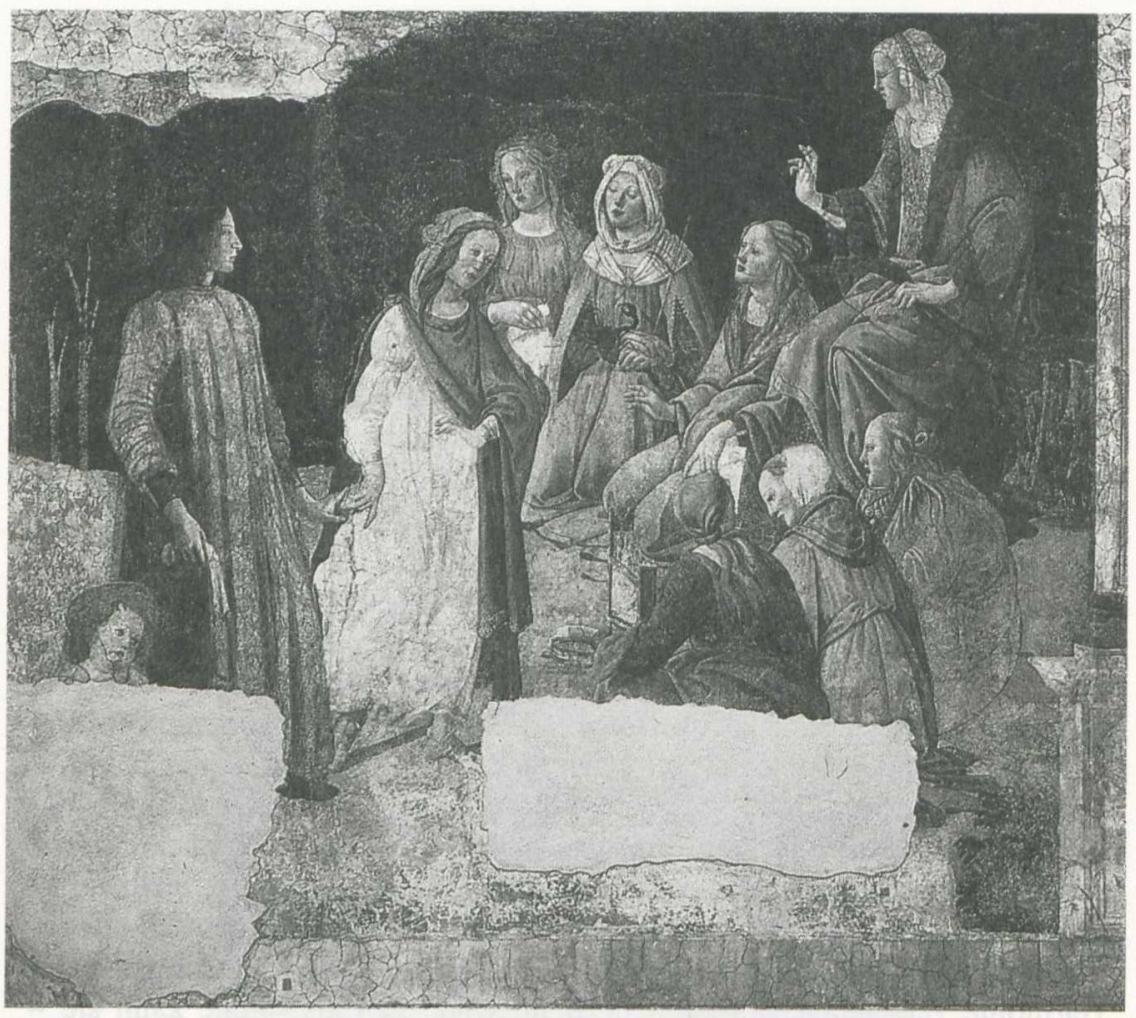

Abb. 8 Sandro Botticelli: Lorenzo Tornabuoni wird von der weiblichen Gestalt Grammatica der Prudentia empfohlen, die von den übrigen freien Künsten umgeben ist. Fragment eines Wandgemäldes, auf Leinwand übertragen. Paris, Musée du Louvre. 


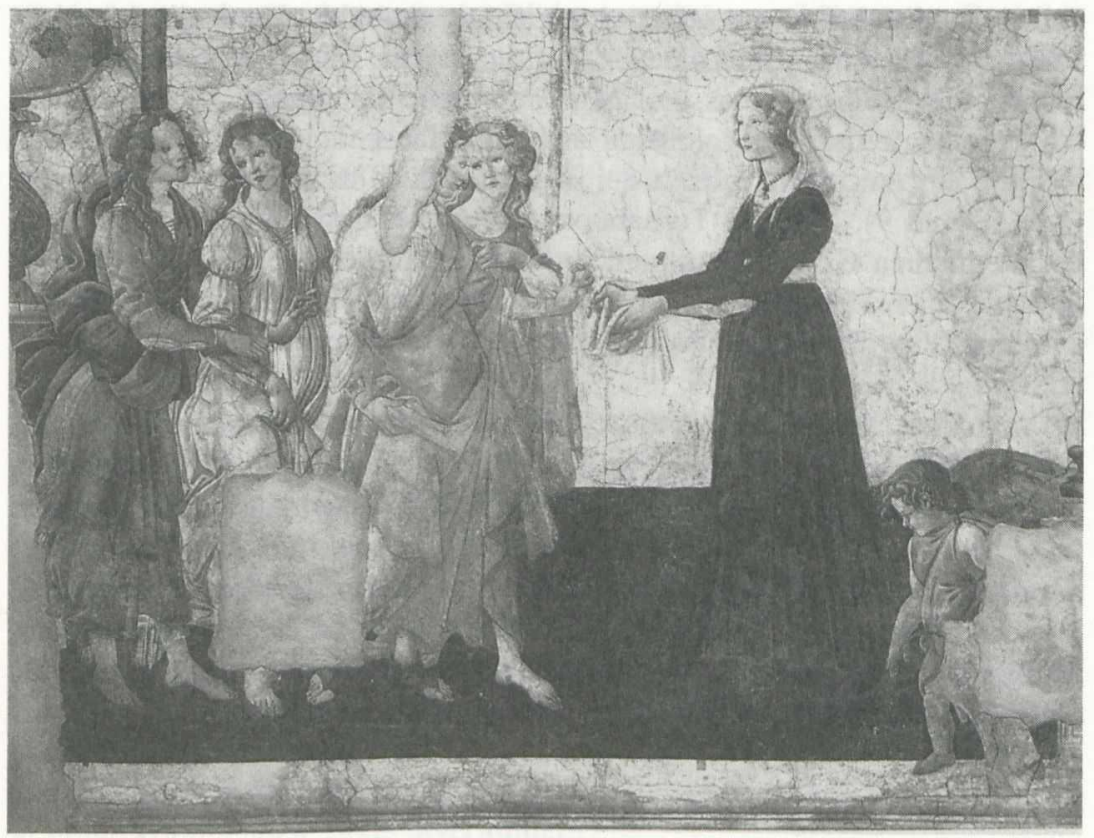

Abb. 9 Sandro Botticelli: Giovanna degli Albizzi empfängt eine Blumengabe von Venus, die von den drei Grazien begleitet wird. Fragment eines Wandgemäldes, auf Leinwand übertragen. Paris, Musée du Louvre. 
Dabei ist die Primavera nicht das einzige Gemälde Botticellis, in dem Martianus Capellas Hochzeit der Philologie für Bilderfindungen genutzt wurde, die Hochzeitliches und Musikalisches implizieren. Davon zeugen die Fragmente der Wandgemälde aus dem Hause Tornabuoni, deren Entstehung eng mit der Hochzeit der Eigentümer 1486 zusammenfallen muß (Abb. 8 und 9). Lorenzo Tornabuoni, ein Onkel des Magnifico, wird von der weiblichen Gestalt Grammatica der Prudentia empfohlen, die von den übrigen freien Künsten umgeben ist. Die Gemahlin, Giovanna degli Albizzi, empfängt eine Blumengabe von Venus, die von den drei Grazien begleitet wird.

Veronika Mertens hat in ihrer 1994 publizierten Dissertation gezeigt, daß die Darstellung der drei Grazien wahrscheinlich von der Schilderung im 2. Buch der Hochzeit der Philologie (132) angeregt wurde, wo die Grazien der Braut ihre Gaben zukommen lassen, um sich dann "mit harmonischen Bewegungen dem Hochzeitstanz anzuschließen « ${ }^{65}$ Aber auch die Darstellung der sieben freien Künste dürfte kaum ohne Anlehnung an Martianus erfolgt sein, der im Quattrocento nach wie vor die Autorität für die septem artes war.

\section{VIII. instauratio}

Eine weitere Grundidee des Bildgedankens der Primavera kann mit dem

- Verb instaurare, das den Aufruf der Venus zur Erneuerung des Festes einleitet, ausgelöst worden sein und ist mit diesem auf den Begriff gebracht. Die substantivierte Form instauratio wurde synonym zu »renovatio, repetitio, rinnovazione... ${ }^{66}$ gebraucht. ${ }^{67}$ Es ist verlockend anzunehmen, daß der Gedanke der instauratio in der Primavera nicht allein auf den Frühling im Sinne der renovatio mundi bezogen wurde und auch nicht allein auf die Erneuerung und Vollendung des Hochzeitsfestes durch Harmonia. Mit der

65 Vgl. Veronika Mertens: Die drei Grazien. Studien zu einem Bildmotiv in der Kunst der Neuzeit. Wiesbaden 1994 (Gratia. Bamberger Schriften zur Renaissanceforschung 24; Diss. phil. Freiburg i. Br. 1994), S. 190-194 (zur Primavera vgl. ebd. S. 184-190); außerdem Martianus Capella, S. 41.

66 Aegidius Forcellini: Lexicon totius Latinitatis. Bd. 2. Patavii 1940, S. 875.

67 Thesaurus linguae Latinae. Bd. 7,1, Sp. 1974-1978. Aus der Vulgata war es zum Beispiel in den Verbindungen minstauratio templi « $(4 \mathrm{Rg} 12,5.7)$ und »instauratio domus Dei« (2 Par 24,27) bekannt. In der klassischen antiken Literatur scheint es sich, wie bei Martianus, besonders auf die Erneuerung und Wiederbelebung von Festen und Spielen bezogen zu haben (Cicero: Harusp. resp. 11,23: Si ludi sunt non rite facti, mentes deorum immortalium, ludorum instauratione, placantur. - Livius: Historiarum lib. 2,36: Ludi forte ex instauratione magni parabantur). - Für den Gebrauch des Wortes in der QuattrocentoDichtung mag ein Zitat des als "Piovano Arlòtto« bekannten Florentiners Arlotto Mainardi (1396-1484) dienen: »Instaurò ... con molta diligenzia le possessioni in farle cultivare, seminare, piantare « ([Piovano Arlotto, 3]. Der Satz entstammt den nach seinem Tod, 1484, kompilierten und dutzendfach neu aufgelegten »Facecie, Piacevoleze, Fabule e Motti«: Motti e facezie del Piovano Arlotto. Hg. von Gianfranco Folena. Neapel 1953). 
Neubelebung antiker Mythologie, Bildungstradition und Kunst im Gemälde wollte der Auftraggeber wahrscheinlich zugleich den eigenen Anteil an der instauratio der Antike überhaupt zur Geltung bringen. In diesem Sinne kann das Frühlings- und Hochzeitsbild zugleich als Paradigma für die Wiederbelebung antiker Kultur und somit als programmatisch für den $\mathrm{Re}$ naissance-Gedanken gelten.

$\mathrm{Da}$ die Primavera zu den wichtigsten Bildzeugnissen für Antikenaneignung und -wiederbelebung des Quattrocento gehört, ist unumstritten. Sollte die hier vertretene These zur Entwicklung des Bildprogramms standhalten, so hieße das allerdings, daß die Art und Weise, in der Botticelli auf Antikes zurückgriff, deutlich den bisher vorherrschenden Thesen zu dieser Frage widerspricht. Seit Aby Warburgs Studie zur Primavera ging man zumeist davon aus, daß die inventio des Bildes durch den mit den mittelalterlichen Traditionen brechenden, direkten Rückgriff auf antike Quellen und durch Anlehnung an die sich auf solche humanistische Studien gründende zeitgenössische Poesie zustande kam. Charles Dempsey hat diese These geradezu zu einer Art Methode erhoben, indem er behauptete, daß der als poetisch vorausgesetzte Charakter der Primavera von vornherein die literarische Gattung und die Qualität (und implizit: die Entstehungszeit) der als Quellen in Frage kommenden Texte determiniere. ${ }^{68}$

Demgegenüber sollte hier gezeigt werden, daß Botticelli oder seine Auftraggeber und Berater für das Programm der Primavera sich für den größten Teil der Bilderfindung auf einen über Jahrhunderte kontinuierlich überlieferten, mittelalterlichen Bildungsstrang stützen konnten, der nun unter gewandelten Gesichtspunkten aktiviert und fruchtbar gemacht wurde. ${ }^{69}$

Ob und inwieweit diese Art der Wiederbelebung der Antike mit der Aneignung antiker Kunst im Bild korrespondiert, wäre noch zu klären. Eine genaue Untersuchung darüber, ob bestimmte Vorbilder der antiken Kunst sei es in Gestalt erhaltener Denkmäler oder literarischer Beschreibungen für die Primavera tatsächlich eine Rolle spielten und auf welche Art und Weise diese vor dem Hintergrund bestehender Darstellungstraditionen Eingang in die Malerei fanden, ist in noch bei weitem nicht befriedigendem $\mathrm{Maß}$ erfolgt.

\section{IX. ut pictura musica}

Unter dem Leitmotiv der Harmonie bietet die Primavera mit Hilfe antiker mythologischer Gestalten eine Allegorie des Frühlings, oder genauer des

68 Dempsey: Portrayal.

69 Dies hätte deutliche Parallelen in Ficinos Platon-Rezeption durch die >Brilleく Plotins oder Pico della Mirandolas Kenntnis heidnischer Mysterien aufgrund spätantiker und mittelalterlicher Quellen: vgl. Wind: Pagan mysteries, S. 24-30. 
Hochzeitsmonats Mai und der mit diesem verknüpften Vorstellung von Fruchtbarkeit, Schönheit und Liebe. In seiner spezifischen Gestaltungsweise ist das Bild - ganz im Sinne von Martianus Capella - als eine Art allegorischer Hochzeits- und Brautzimmerschmuck zu verstehen.

Die Verbindung der Bildidee zum Ehelager und damit konkret zum lettuccio in Lorenzo di Pierfrancescos Haus ist in der Hochzeit der Philologie mit den Begriffen thalamus und fulcrum gleich zweifach nahegelegt. Thalamus steht übrigens für das Ehebett ebenso wie für das Frauengemach. Die Eheleute könnten somit die von Venus dirigierte Erneuerung des Hochzeitlichen als Anregung zur Erneuerung der Hochzeitsnacht verstanden haben, für die die Frühlingsgöttin immer neu das Lager schmückt.

Botticelli hat, ausgehend von antiken Traditionen, mit den Mitteln der bildenden Kunst nach den Regeln der Harmonie gleichsam das Urbild eines hochzeitlichen Tanzes komponiert und damit die Malerei in den Rang einer freien Kunst erhoben. Er zeigt, daß die Malerei nicht allein der praktizierten Musik vergleichbar ist, sondern auch dazu befähigt, aus der Kenntnis der Gesetzmäßigkeit der Harmonie anspruchsvolle Bildkompositionen zu schaffen. Dem Horazischen ut pictura poesis, das sich damals im Paragone besonderer Beliebtheit erfreute, stellte Botticelli ein ut pictura musica zur Seite, das erst im 16. und 17. Jahrhundert in der Kunstliteratur ausführlicher begründet wurde. ${ }^{70} \mathrm{Da} \beta$ er dabei auch den Vergleich zur Poesie nicht scheuen mußte, zeigt die ungewöhnliche Bildidee selbst, mit der nicht einfach eine bestimmte poetische Äußerung in eine bildliche übersetzt wurde; vielmehr wurden bestimmte, von den Versen eines spätantiken Autors nahegelegte Vorstellungen in eine eigenständige Allegorie verwandelt. Wenn sich auch deren Inhalte mit Themen des Florentiner Neuplatonismus überschneiden, spricht meines Erachtens nichts dafür, das Bild selbst als primär philosophische Äußerung zu betrachten, wie dies oft getan wird. Mit dem Neuplatonismus gemeinsam ist der Primavera allerdings zumindest die

70 Vgl. zum Beispiel Bernard Teyssèdre: Peinture et musique: La notion d'harmonie des couleurs au XVIIe siècle français. In: Stil und Überlieferung in der Kunst des Abendlandes. Akten des 21. Internationalen Kongresses für Kunstgeschichte in Bonn 1964. Bd. 3. Berlin 1967, S. 206-214. - Pavel Preiss: Farbe und Klang in der Theorie und Praxis des Manierismus. In: Colloquium musica bohemica et europea (Brno 1970), 5th International Musical Festival, Brünn 1970. Arranged and edited by Rudolf Pecman. Chairman of the colloquium Jiri Vyslovzil. Brno 1972, S. 163-170. - Ulrike Groos: Ars Musica in Venedig im 16. Jahrhundert. Hildesheim, Zürich, New York 1996 (Diss. phil. Münster 1994) (Studien zur Kunstgeschichte 108). - Zum Verhältnis einzelner italienischer Renaissance-Künstler zur Musik vgl. etwa Marzia Faietti: Protoclassicismo e cultura umanistica nei disegni di Francesco Francia. In: Il Classicismo. Medioevo, Rinascimento, Barocco. Atti del Colloquio Cesare Gnudi (Bologna 1986). Bologna 1993, S. 171-192, hier S. 183-184. - Kürzlich wurde gezeigt, daß Albrecht Dürers Interesse an der menschlichen Proportion mit musikalischen Überlegungen einherging, die zur Komposition eines Orgelstücks (ca. 1512/13) führten: Hans Herrmann Schmid: Dürer als Komponist eines »Salve Regina« für Orgel. In: Literatur in Bayern 29 (München 1992). 
Absicht der Inspiration, die offenbar mit diesem - gerade nicht didaktisch erscheinenden - Bild verbunden war.

$\mathrm{Da}$ Botticelli dennoch weitere, hier nicht berücksichtigte Bildaussagen getroffen haben mag, wird nicht bestritten; ebensowenig, daß das Bildprogramm von weiterreichenden Überlegungen mitbestimmt sein oder solche zumindest bei Zeitgenossen angeregt haben kann. Auf der hier vorrangig interessierenden Ebene der Bilderfindung haben zum Beispiel sicher literarische Beschreibungen antiker Bilder, wie Alberti sie Künstlern seiner Zeit als vorbildliche Beispiele für gelungene inventiones anempfohl, oder Schilderungen klassischer Autoren, die bildliche Vorstellungen evozieren, für die weitere Gestaltung der Primavera eine Rolle gespielt. So wird die von Alberti aufgegriffene Beschreibung der drei Grazien von Seneca sicher nicht zu unrecht für eine Quelle zur Gestaltung der Grazien Botticellis gehalten. ${ }^{71}$ Erst zur Untersuchung dieses Schritts der inventio, der Umsetzung einzelner Figuren oder Gruppen des Bildprogramms in eine konkrete ikonographische Erscheinungsform, scheint es angebracht, über die Hochzeit der Philologie hinaus weitere mögliche Textquellen zum Verständnis des Bildes zu befragen. Daraus ergäbe sich ein zusätzlicher Aspekt des $u t$ pictura poesis, wie Botticelli ihn auch mit der Verleumdung des Apelles verwirklichte. ${ }^{72}$ Die von Lukian gebotene Beschreibung dieses Gemäldes des Apelles wurde, ausgehend von ihrer Wiedergabe in Albertis Malereitraktat, zur wohl bedeutendsten Ekphrasis für die Renaissancemalerei. ${ }^{73}$

Über solche literarischen Bezüge hinaus legen aber auch die im Bild vorgestellten Handlungen und die Bezüge der Figuren untereinander weiterreichende Aussagen nahe.

Hier allerdings sollte lediglich untersucht werden, welche Gedanken zum Grundkonzept, zum ersten Schritt der inventio dieses außergewöhnlichen Bildes geführt haben können.

71 Vgl. Seneca: De beneficiis, I, 3; Alberti: De pictura, Liber III, 54., S. 97; vgl. auch Lightbown, S. 130-133; Mertens: Grazien, S. 31f., S. 66-72.

72 Florenz, Galleria degli Uffizi, ca. 1494/95, $62 \times 91 \mathrm{~cm}$.

73 Alberti: De Pictura (ed. Grayson), Liber III, 53., S. 94, 96. Vgl. dazu Jean-Michel Massing: Du texte à l'image. La calomnie d'Apelle et son iconographie. Straßburg 1990. 